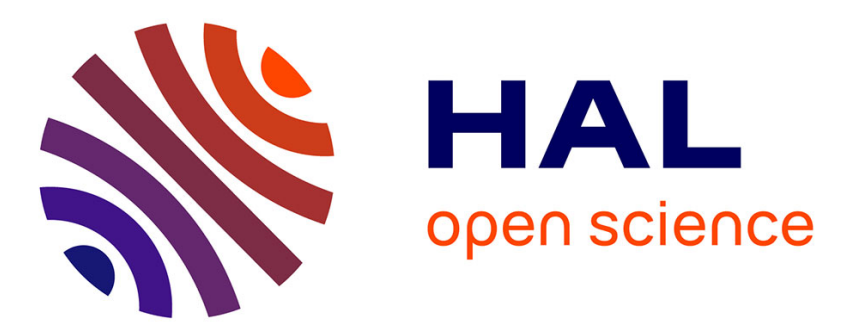

\title{
Assessment of pedestrian discomfort glare from urban LED lighting
}

Céline Villa, Roland Bremond, Enoch Saint Jacques

\section{To cite this version:}

Céline Villa, Roland Bremond, Enoch Saint Jacques. Assessment of pedestrian discomfort glare from urban LED lighting. Lighting research and technology, 2016, 59p. 10.1177/1477153516673402 . hal01381250

\author{
HAL Id: hal-01381250 \\ https://hal.science/hal-01381250
}

Submitted on 14 Oct 2016

HAL is a multi-disciplinary open access archive for the deposit and dissemination of scientific research documents, whether they are published or not. The documents may come from teaching and research institutions in France or abroad, or from public or private research centers.
L'archive ouverte pluridisciplinaire HAL, est destinée au dépôt et à la diffusion de documents scientifiques de niveau recherche, publiés ou non, émanant des établissements d'enseignement et de recherche français ou étrangers, des laboratoires publics ou privés. 


\section{Assessment of pedestrian discomfort glare from urban LED lighting}

\section{Villa $\mathrm{PhD}$, R Bremond $\mathrm{PhD}$ and E Saint-Jacques MSc}

Laboratoire Exploitation, Perception, Simulateurs et Simulations (LEPSIS). Institut Francais des Sciences et Technologies des Transports, de l'Amenagement et des Reseaux (IFSTAAR), Cite Descartes, Champs-sur Marne, France

Short title: Discomfort glare experienced by pedestrians

Received 1 July 2016; Revised 7 September 2016; Accepted

Due to different visual tasks and gaze patterns, the discomfort glare experienced by pedestrians may differ from that experienced by drivers. This paper investigates the discomfort glare experienced by pedestrians under various urban LED luminaires through psychovisual experiments conducted on a test track. The ability of state-of-the-art models to predict the level of discomfort glare, measured on the de Boer rating scale, for this application is also investigated. With one exception, the models all overestimate the mean subjective discomfort glare compared to the experimental data. Models proposed by Lin et al (2015) and Bullough et al $(2008,2011)$ perform well. However, the implementation of these models is not straightforward because choices are needed to estimate some of the variables such as the background luminance and the glare source area. 
Address for correspondence: Celine Villa, IFSTTAR/LEPSIS, Cite Descartes, 20 boulevard Newton, 77447 Champ-sur-Marne, France

E-mail: celine.villa@ifsttar.fr

\section{Nomenclature}

$\mathrm{I}_{80}$ : luminous intensity at $80^{\circ}$ from the vertical (in cd)

$\mathrm{I}_{88}$ : luminous intensity at $88^{\circ}$ from the vertical (in cd)

$\mathrm{F}$ : luminous surface of the luminaire seen at $80^{\circ}$ from the vertical $\left(\right.$ in $\left.\mathrm{m}^{2}\right)$

h' : adjusted luminaire height (luminaire height - 1.5m (observer's eye height)) (in m)

$\mathrm{p}$ : number of poles per kilometre

$\theta$ : eccentricity, i.e. the angle between the line of sight and the light source (in arc minute in Sch74, in degrees in the other models)

$\mathrm{L}_{\mathrm{a}}:$ adaptation luminance (in $\mathrm{cd} / \mathrm{m}^{2}$ )

$\mathrm{L}_{\mathrm{b}}$ : background luminance (in $\mathrm{cd} / \mathrm{m}^{2}$ )

$\mathrm{L}_{1}:$ source luminance (in $\left.\mathrm{cd} / \mathrm{m}^{2}\right)$

$\mathrm{E}_{1}$ : vertical illuminance due to the source (in $1 \mathrm{x}$ )

$E_{s}$ : vertical illuminance due to the immediate surround of the source (in lx)

$\mathrm{E}_{\mathrm{a}}$ : vertical ambient illuminance (in $\mathrm{lx}$ )

$\mathrm{E}_{\mathrm{b}}$ : vertical illuminance due to the background (in lx) 
$\omega:$ solid angle from the observer (in sr)

$\mathrm{E}_{\text {baffle: }}$ vertical illuminance measured when the illuminance meter is covered with a circular baffle to remove the direct illuminance from the source (in lx)

$\mathrm{E}_{\mathrm{Lantern}}$ : vertical illuminance due to the lantern (in lx)

$\mathrm{E}_{\mathrm{LED}}$ : vertical illuminance due to the LED module (in $1 \mathrm{x}$ )

$\mathrm{L}_{\text {Lantern: }}$ average vertical luminance of the lantern (in $\left.\mathrm{cd} / \mathrm{m}^{2}\right)$

$\mathrm{L}_{\mathrm{LED}}$ : average vertical luminance of the LED module (in $\mathrm{cd} / \mathrm{m}^{2}$ )

$\mathrm{L}_{\max }:$ maximum luminance of the source (in $\mathrm{cd} / \mathrm{m}^{2}$ )

$\mathrm{L}_{\mathrm{RZ}}$ : average luminance of the road zone (in $\mathrm{cd} / \mathrm{m}^{2}$ )

$\mathrm{L}_{\mathrm{DZ}}$ : average luminance of the disc zone ( $30^{\circ}$ circular zone) (in $\left.\mathrm{cd} / \mathrm{m}^{2}\right)$

$\mathrm{E}_{\mathrm{RZ}}$ : vertical illuminance computed from $\mathrm{L}_{\mathrm{RZ}}$ (in $\left.1 \mathrm{x}\right)$

$\mathrm{E}_{\mathrm{DZ}}$ : vertical illuminance computed from $\mathrm{L}_{\mathrm{DZ}}$ (in $\left.1 \mathrm{x}\right)$

$\mathrm{L}_{\text {black: }}$ average luminance of the whole image captured when all the lanterns are switched off (in $\left.\mathrm{cd} / \mathrm{m}^{2}\right)$

$\mathrm{E}_{\text {black }}$ : vertical illuminance computed from $\mathrm{L}_{\text {black }}($ in $1 \mathrm{x}$ ) 


\section{Introduction}

\subsection{Context}

Urban lighting is designed for all road users, including pedestrians. As far as pedestrians are concerned, the main factors influencing lighting quality are feeling of personal security, facial recognition and visibility ${ }^{1}$. However, the performance of a lighting installation must be balanced against its costs in terms of financial cost, energy consumption, light pollution and glare. Nowadays, pedestrian comfort has become a research topic ${ }^{2-5}$, especially with the increasing use of LED luminaires. Indeed, the small size of LED chips can lead to high luminances that may produce discomfort glare ${ }^{6}$. Kostic et al. conducted a field experiment to compare subjective judgments on LED and metal halide lighting with respect to various criteria about the light quality (illuminance, colour) and pedestrians' experience (security, comfort $)^{2}$. Discomfort glare was evaluated on a 5-point scale while walking along the path, looking straight ahead. Luo et $a .^{3}$ studied pedestrian zone lighting by collecting preference judgments and eye fixations from five participants, but no glare was reported by the participants in this experiment. Miller et al. ${ }^{4,5}$ investigated pedestrian-friendly outdoor lighting. To avoid pedestrian glare, the authors recommended limiting the maximum luminance, and avoiding strong luminous intensity / luminance variations with the viewing angle. They advised using frosted and diffusing optics to avoid a direct view of the LEDs. In addition, they raised methodological problems for discomfort glare assessment, including the photometric description of the scene (source size, background area definition). They also highlighted the need for new investigations on pedestrian discomfort glare, especially about the influence of the luminance distribution, the number of sources and the temporal changes occurring as the pedestrian walks along the path. 
There is no consensus in previous work about the effect of the number of sources on discomfort glare. Schmidt Clausen and Bindels ${ }^{7}$ tested multiple glare sources very close to each other at low eccentricities (i.e. small angle between the line of sight of the driver and the light sources) and predicted that discomfort glare increases with the number of sources (additivity). In assessing road lighting installations, de Boer and Schreuder ${ }^{8}$ found an increase of +0.5 on the de Boer scale when the number of visible light sources was about halved (from 11 to 6 luminaires) for a driver point of view. Bullough et al. ${ }^{9}$ addressed outdoor lighting independently of the participant's status (driver or pedestrian) and found no effect of a second distant source, when this source is at least 9 degrees away from the line of sight. Zhu et al. ${ }^{10}$ found no effect of the number of sources on discomfort glare either; they used a vertical panel of LEDs to simulate road lighting in the laboratory. Pedestrian studies also suggest that observers feel the discomfort glare due to one luminaire at a time ${ }^{5}$, and judge the lighting quality with respect to the luminaire closest to them ${ }^{11}$.

In order to quantify the quality of a lighting installation in terms of comfort, one would like to predict the discomfort glare level. For that purpose, various models ${ }^{7-9,12-14}$ have been developed mostly in laboratories (i.e. with simplified and controlled visual scene photometry) to predict discomfort glare from automotive $\mathrm{e}^{7}$ and/or outdoor lighting ${ }^{9,12-14}$. These models predict the discomfort glare level on the de Boer scale ${ }^{8}$ from the photometric and geometric characteristics of the visual scene. These models estimate the discomfort glare experienced by a static observer, with a fixed gaze direction.

As pointed out by Miller et al..$^{5}$, discomfort glare is somehow different for pedestrians compared to drivers. Although pedestrians and drivers share the same visual system, their 
visual experience (including glare) differ in some respect. Firstly, they do not perform the same visual tasks, which is known to impact visual performance ${ }^{15}$ as well as discomfort glare $^{16}$. Secondly, their regions of interest (in which information to perform the task is collected) are different; Driver gaze has been studied intensively in the last 20 years $^{17,18}$, while pedestrian gaze allocation still is in its infancy ${ }^{19}$. This impacts both the source eccentricity (which in turn impacts glare) and the adaptation luminance, which is a proxy for the luminance in the area where people stare, that is, the Area of Interest (AoI). Also, a typical driver gaze is much more constrained to look towards specific AoI (tangent point, vanishing point, other road users, etc.) than a pedestrian's gaze, which is expected to increase the discomfort glare $^{20}$. Another issue is that some level of overhead glare is experienced by pedestrians, not by drivers, because of the cut-off of the car roof. Finally, the speed of movement is different, and dynamic effects on glare may be different.

The models for predicting discomfort glare level have each been developed using data for a given range of variables (see Table 1). Many of these, especially the range of tested eccentricities, do not cover all the values appropriate for a pedestrian situation. In addition, these models have not been used so far for outdoor lighting from a pedestrian point of view, and therefore deserve to be assessed in such a scenario.

\subsection{Discomfort glare models}

Previous work on lighting design investigated the link between geometric and photometric characteristics of the illuminated scene and discomfort glare. The main photometric/geometric factors identified in the literature are the source luminance, the background or adaptation luminance ${ }^{21}$, the vertical illuminance at the eye of the observer ${ }^{22}$, 
the source eccentricity (angle between the observer's gaze direction and the source) and the solid angle of the source from the observer point of view. Based on these factors, various models have been proposed ${ }^{7,9,12-14,23}$. In outdoor lighting, most of them predict a level of discomfort glare on a subjective scale, such as the de Boer scale ${ }^{8}$. The de Boer scale has been the most widely used scale in previous outdoor lighting studies ${ }^{7-10,13,14,21,22,24-26}$ and therefore was implemented in our experiment in order to compare predictions from different models to our experimental data. This is why our overview below only focuses on the models predicting a de Boer rating. The ranges of values of the variables employed in the original papers to fit each of these models are shown in Table 1. A more comprehensive review of discomfort glare models was conducted by Gibbons and Edwards ${ }^{27}$.

De Boer and Schreuder ${ }^{8}$ introduced a model to determine the level of discomfort glare in road lighting installations using the de Boer scale: the Glare Control Mark (GCM) index ${ }^{8,28}$. The model was developed with data collected from participants who judged the discomfort glare from a driver's point of view moving along a $300 \mathrm{~m}$ lighting installation, simulated on a 1:50 scale dynamic mock-up. It accounts for the light distribution (cut-off) by means of luminous intensity levels in two directions $\left(80^{\circ}\right.$ and $88^{\circ}$ from the vertical), the luminous surface, the average road luminance, and the number of sources (height and pole spacing), see Equation 1. In this experiment, the authors found no impact of the nonuniformity of the road luminance on discomfort glare.

$$
\begin{aligned}
\mathrm{GCM}=13.84 & -3.31 \log \mathrm{I}_{80}+1.3\left(\log \frac{\mathrm{I}_{80}}{\mathrm{I}_{88}}\right)^{0.5}-0.08 \log \frac{\mathrm{I}_{80}}{\mathrm{I}_{88}} \\
+ & 1.29 \log \mathrm{F}+0.97 \log \mathrm{L}_{\mathrm{b}}+4.41 \log \mathrm{h}^{\prime}-1.45 \log \mathrm{p}
\end{aligned}
$$


Schmidt-Clausen and Bindels ${ }^{7}$ introduced a model to predict the discomfort glare on the de Boer scale due to automotive lighting (the model is hereafter referred to as Sch74). It takes as input the source vertical illuminance at the observer's eye and the adaptation luminance (which is taken as the average luminance of the road surface); see Equation 2. As in the GCM model, the adaptation luminance is not easy to estimate. Moreover, as in all models with source eccentricity, glare is undefined when looking directly towards the light source $(-\infty$ on the de Boer scale).

$\operatorname{Sch} 74=5.0-2.0 \log _{10}\left(\sum_{\mathrm{i}} \frac{\mathrm{E}_{\mathrm{li}}}{0.003 \times\left(1+\sqrt{\frac{\mathrm{La}_{\mathrm{a}}}{0.04}} \theta_{\mathrm{i}}^{0.46}\right.}\right)$

In order to overcome these limitations, Bullough et al. ${ }^{9}$ introduced an index for outdoor lighting, predicting the discomfort glare on the de Boer scale from three vertical illuminance levels at the observer's eye (the model is hereafter referred to as Bul08), when looking directly at the source. These illuminance levels are respectively associated to the observed light source $\left(E_{1}\right)$, the surround $\left(E_{s}\right)$, and the ambient term $\left(E_{a}\right)$, (see DG in Equation 3); the total vertical illuminance $E_{\text {tot }}$ at the observer's eye is the sum of $E_{l}, E_{s}$ and $E_{a}$. To develop their model, Bullough et al. carried out a set of experiments in the laboratory and outdoor with metal halide or mercury vapour lamps in front of the observers. Later, Bullough et al. ${ }^{12}$ highlighted the limits of their previous model and proposed a model extension (Bul11 in Equation 3), which includes as input the source luminance $\mathrm{L}_{1}$ when the source subtends more than $0.3^{\circ}$ of visual angle (the model is hereafter referred to as Bul11). 


$$
\left\{\begin{array}{c}
D G=\log _{10}\left(E_{l}+E_{s}\right)+0.6 \log _{10}\left(\frac{E_{1}}{E_{s}}\right)-0.5 \log _{10}\left(E_{a}\right) \\
\text { Bul08 }=6.6-6.4 \log _{10}(D G) \\
\text { Bul11 }=6.6-6.4 \log _{10}(D G)+1.4 \log _{10}\left(\frac{50000}{L_{l}}\right)
\end{array}\right.
$$

More recently, Lin et al. ${ }^{13}$ introduced a model derived from laboratory data obtained with a $3000 \mathrm{~K}$ LED source located $2 \mathrm{~m}$ ahead of the observers (the model is hereafter referred to as Lin14, see Equation 4). The model was also tested in the laboratory with a $6500 \mathrm{~K}$ LED source and outdoors with a $5000 \mathrm{~K}$ LED source. The authors found an offset in average judgments related to the correlated colour temperature, the discomfort glare being higher for higher correlated colour temperatures, but they did not introduce this factor in their model.

$$
\operatorname{Lin} 14=3.45-\log _{10}\left(\frac{\left(\mathrm{L}_{\mathrm{l}} \omega\right)^{2.21}}{\mathrm{Lb}_{\mathrm{b}}^{1.02} \theta^{1.62}}\right)
$$

Lin et al. ${ }^{14}$ proposed another model in 2015 (the model is hereafter referred to as Lin 15, see Equation 5). The Lin15 model is based on the illuminance due to the source and background illuminance. Unlike Lin14, the luminance and the solid angle of the source are not needed. Moreover, the Lin15 model was based on data collected with a larger range of background photometry (0, 10 and 2001x) than the Lin 14 model (around $\left.1-10 \mathrm{~cd} / \mathrm{m}^{2}\right)$, see Table 1 . The main difference between these models is the value of the constant which allows for rescaling the log magnitude to the de Boer scale (3.45 in Lin14 vs 7.09 in Lin15): Lin14 predicts higher discomfort glare than Lin15.

$$
\operatorname{Lin} 15=7.09-\log _{10}\left(\frac{E_{1}^{2.21}}{E_{b^{1.02}} \theta^{1.62}}\right)
$$




\subsection{Model implementation: State of the art}

These models were mostly developed in laboratory conditions with uniform backgrounds and light sources. In heterogeneous real-world scenes, evaluating luminance values requires some choices. Previous work assessing these models ${ }^{13,29}$ has been done by laboratory experiments and followed the method described in the original papers. Very few studies have implemented these models outdoors ${ }^{30,31}$.

\subsubsection{Background luminance}

When the visual scene is complex, the background luminance depends on the geometric definition of the background area and on the computational method employed to reduce a luminance distribution into a single luminance value. In previous work $^{7,32}$, the background luminance is often defined as the average luminance of the road surface. However, drivers and pedestrians look at specific and different AoI. The adaptation luminance roughly reflects the luminance in these areas of interest, and thus they are expected to be different. More specifically, the AoI of pedestrians is expected to be larger, and farther away from the light sources and their gaze is expected to be less constrained ${ }^{20}$. Researches are in progress $s^{3,34}$ to understand the shape, size and location of each road user's adaptation $\operatorname{area}^{33,35-37}$, and some researches focus on pedestrians, investigating pedestrians gaze patterns with eye-trackers ${ }^{3,33,34,37}$. Davoudian et al. found that pedestrians spend 40-50\% of the time looking at the footpaths ${ }^{34}$. Recently, Uchida et al. introduced a simulation method to compute peripheral adaptation luminance in an area of measurement (AOM) in mesopic outdoor scenes, from a static luminance distribution and an eye movement distribution ${ }^{35}$. The authors 
tested various AOM sizes on the road surface with drivers' or pedestrians' eye movement distributions, and they compared the simulation with simpler proxies, such as the average luminance in the AOM and the average luminance in the whole image. They found that the adaptation luminance could be predicted with the average luminance in the AOM, especially when eye movements are not large. For pedestrians, the footpath surface average luminance is a fair predictor of the adaptation luminance, except when many high luminance sources are in the surroundings. Cengiz et al. assumed a disc-shaped visual adaptation field, centred on the mean gaze direction ${ }^{33}$. They computed the average luminance in circular fields extending $1^{\circ}$ to $20^{\circ}$ from the line of sight of drivers. Maksimainen et al. compared average mesopic luminance values in the AOM, with various sizes and shapes (road surface, circular, elliptical). For each street illustrations employed in this work, they obtained a standard deviation of the average luminances computed in various AOM around $21 \%^{36}$. To implement discomfort glare models for pedestrian applications, Kohko et al. tested averaging the luminance on the road surface and in a circular visual field $\left(20^{\circ}\right.$ or $\left.40^{\circ}\right)$, "but none of them showed acceptable fitting, ${ }^{30}$. Finally, they used a constant background luminance for all stimuli.

In some models ${ }^{7,14}$, the background photometry is measured by switching off the light source. However, in a pedestrian case study, urban lighting contributes to both glare and adaptation (indirect lighting). Therefore, in such a case study, the indirect component of the sources needs to be taken into account in the background term estimation.

\subsubsection{Light source photometry}


Some models use the source vertical illuminance as input. In this approach, the whole lantern defines the light source. The larger the source region, the higher the illuminance and therefore the discomfort glare.

Other models use the product of the source luminance and its solid angle as input, but they do not specify the region which should be considered and how to compute luminance and solid angle of a LED source. Indeed, LED light sources can be non-uniform compared to other common sources (e.g. High Pressure Sodium, Metal-Halide, Incandescent, Fluorescent) because they associate many very small sources (LED chips) on a LED module, fitted with optical components. The non-uniformity of LED luminaires may contribute to discomfort glare $^{6,38-40}$. From a luminance map, a computational method has to be chosen to provide a single value as input to the models. In most previous work, the average luminance is used as the input value for the source luminance. The average may not be the most relevant way to estimate the luminance of a non-uniform source; other estimates may also be considered, such as the maximum luminance or a weighted $\operatorname{sum}^{40}$. In addition, whatever the model, the predicted discomfort glare increases with the source luminance as well as with the source solid angle. Therefore, in some cases, model predictions are sensitive to the geometric definition of the light source in an unpredictable way. For example, in urban LED lanterns, the LED module is usually surrounded by optical components (e.g. clear glass, frosted glass). In this case, the source area may be described in at least two ways: either the whole lantern or restricted to the LED module (see Figure 3(b)). The average luminance is higher in the LED module area than in the Lantern area, whereas the solid angle of the LED module area is lower than the solid angle of the lantern area. 


\subsubsection{Implementation of Bullough's model}

This model addresses the discomfort glare from each light source in the lighting installation. Bullough's model needs as inputs the direct source illuminance $\mathrm{E}_{\mathrm{l}}$, the illuminance from the area surrounding the source $E_{s}$ and the ambient illuminance $E_{a} . E_{a}$ is the vertical illuminance measured when the sources are switched off. $E_{1}$ cannot be measured directly. Bullough et al. ${ }^{9,31}$ proposed to deduce $\mathrm{E}_{1}$ from the total vertical illuminance and the vertical illuminance measured when the direct component of the source is hidden from the illuminance meter. It is not possible to "cover" the light source up a $4 \mathrm{~m}$ high pole as proposed by Bullough et al. ${ }^{9}$ in a laboratory setup. This is why, Brons et al. ${ }^{31}$ proposed to shield the illuminance meter with a circular baffle, to remove the direct illuminance of the source $\mathrm{E}_{1}$ from the measurement and deduce it by computation: $\mathrm{E}_{\mathrm{l}}=\mathrm{E}_{\mathrm{tot}}-\mathrm{E}_{\text {baffle }}$.

The illuminance from the area surrounding the source $\mathrm{E}_{\mathrm{s}}$ is not easy to define in a complex scene. It is defined by Bullough et al. ${ }^{9}$ as the remainder found when subtracting $\left(\mathrm{E}_{1}+\right.$ $\left.E_{a}\right)$ from $E_{\text {tot }}$. For pedestrian applications, the same authors ${ }^{31}$ defined the surrounding illuminance $\mathrm{E}_{\mathrm{s}}$ as the difference between the illuminance measured when the lighting installation is switched on except the tested luminaire, and the sum of the direct illuminance levels from all other luminaires $\left(\mathrm{E}_{\mathrm{s}}\right.$ includes the ambient illuminance, unlike Bullough's (2008) definition $\left.{ }^{9}\right)$.

Whatever the computational definition, we understand that the surrounding illuminance is introduced in the model to quantify the adaptation illuminance due to the indirect component of the lighting installation.

\subsubsection{Decomposition of direct and indirect contributions from the light source}


We have seen above that models based on illuminance (i.e. Sch74, Bul08, Lin15) ${ }^{7,9,14}$ require the estimation of the direct illuminance from the light source, as well as the indirect illuminance due to light reflections in the environment. Unfortunately, measuring the vertical illuminance at the observer's eye merges these variables. The measurement can be split into two parts, for example using a baffle on the illuminance meter ${ }^{31}$.

The two components of the vertical illuminance may also be computed from luminance values. Indeed, the direct illuminance due to any region of a luminance map, captured with an imaging luminance measurement device (ILMD), can be calculated from pixel luminance, solid angle and eccentricity, assuming that the gaze is at the centre of the map.

Finally, the background vertical illuminance from a given region may also be estimated from the average luminance over this region assuming the reflecting surfaces to be Lambertian. This assumption is supported by de Boer, who found no influence of the road luminance non-uniformity ${ }^{8}$ and a direct correlation between the average illuminance and average luminance of a lighting installation ${ }^{41}$.

\subsection{Objectives}

A pilot outdoor study was conducted to investigate pedestrian discomfort glare in complex visual scenes (as opposed to controlled laboratory experiments with uniform background and source luminance). The objectives of this work were twofold:

1) To investigate a protocol for assessing the pedestrian discomfort glare from LED urban lighting; 
2) To implement and test the relevance of previously developed models for predicting the pedestrian discomfort glare from LED urban lighting.

The second objective was limited to those models which predict a score on the de Boer scale.

Two experimental protocols were tested. The "Static" protocol involved collecting judgments under various lighting installations, from specific positions and gaze directions. The "Dynamic" protocol involved collecting a global judgment of discomfort glare after walking under the poles. This protocol integrates temporal variations in the glare experience during the walk. Installations were judged with one or two luminaires switched on in order to study the influence of the number of sources. In order to collect robust data, some variability was introduced in the visual environment by using four different lanterns. Viewpoints and gaze directions were also varied.

Mean glare ratings were compared to various model predictions in order to evaluate their relevance for a pedestrian application. This comparison was based on the Root Mean Square Error (RMSE) and on the Spearman correlation $\left(\mathrm{R}^{2}\right)$. RMSE quantifies a global error between the participants' mean ratings and the model predictions. $\mathrm{R}^{2}$ quantifies the ability of the model to predict ordinal results (ranking).

The remainder of the paper is organized as follows. Section 2 is dedicated to the material and methods of our experiment and to the implementation issues of the various models. Section 3 presents the psychovisual results. Section 4 presents the evaluation of each predictive model for a pedestrian application. Findings are discussed in Section 5 while conclusions and future work are presented in Section 6. 


\section{Material and methods}

A psychovisual experiment was carried out on a test track in Les Andelys, France (see Figure

1) in order to collect discomfort glare ratings (on the de Boer scale) under different LED urban lighting installations. The street section was approximately $100 \mathrm{~m}$ long and $5.5 \mathrm{~m}$ wide. The road surface reflection properties was classified according to the CIE standards ${ }^{42}$ : the road surface was class R3 (with a lightness of $\left.\mathrm{Q}_{0}=0.07\right)^{42}$ and $\mathrm{W} 3\left(\mathrm{Q}_{0}=0.02\right)$ for a wet road.
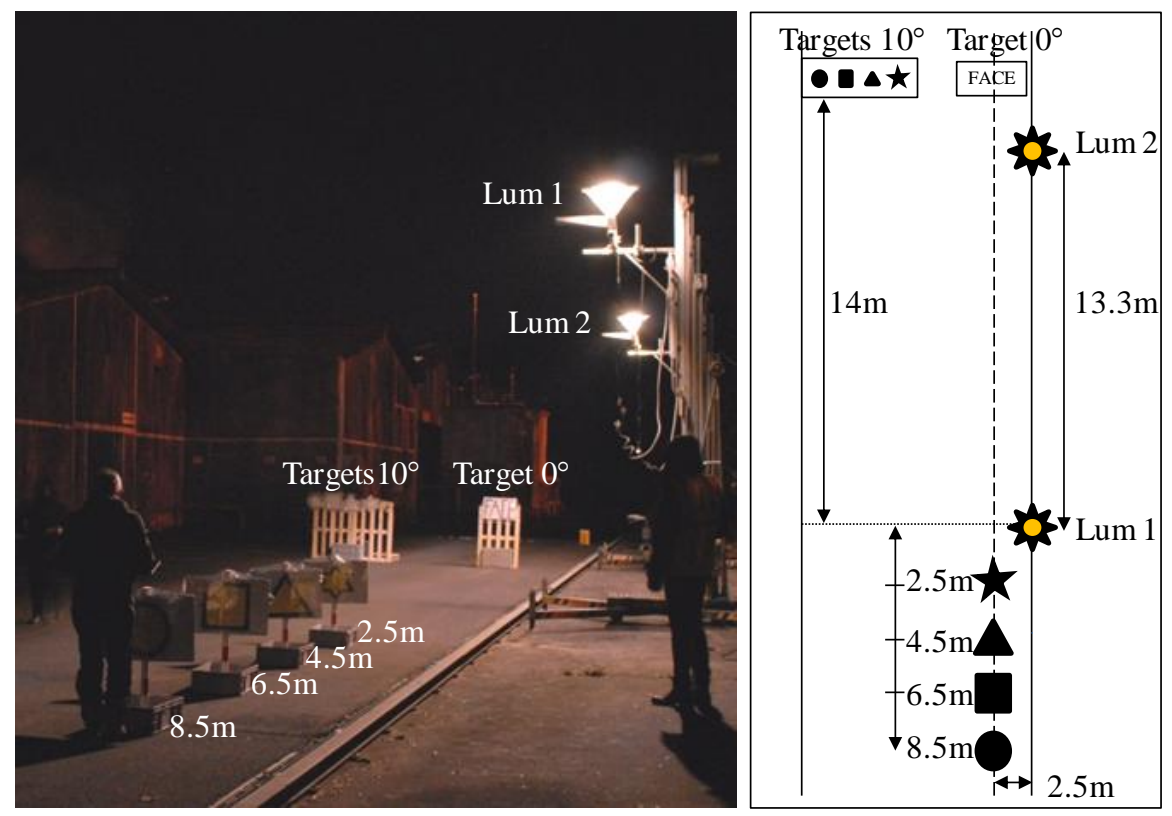

Figure 1. Experimental setup with both luminaires switched on (left) and illustration of the experimental settings (right). The geometrical shapes (circle, square, triangle, star) correspond to the viewing positions in the "Static" protocol; targets $0^{\circ}$ and $10^{\circ}$ are used to control the participants' gaze direction.

\subsection{Description of the urban lighting installations}


The main photometric and geometric characteristics of the lighting installations are provided in Table 2. The spacing between the poles was $13.3 \mathrm{~m}$ and the height of the luminaires was $4 \mathrm{~m}$, which is consistent with the European standard NF-EN13201 ${ }^{43}$ (type S2) (according to DIALUX ${ }^{\mathrm{a}}$ computations). Urban lighting poles are usually 3 to $8 \mathrm{~m}$ in height, and the spacing is defined in order to ensure an average illuminance of between 10 and $15 \mathrm{~lx}$ and a uniformity level $\mathrm{U}_{0}$ (i.e. Minimum/Average) higher than 0.4 on the road. Four lanterns were tested (A, B, C and D in the following). The correlated colour temperature was $4000 \mathrm{~K}$ and the CRI $\mathrm{Ra}=70$ for all lanterns. The luminous flux was chosen so as to ensure similar average horizontal illuminance under each installation.

\subsection{Panel}

Thirty-three participants were involved in this study (14 men, 19 women), with ages between 21 and 60 years $(M=42.2, S D=13.7)$. Their vision was tested with an ErgoVision device (Essilor) prior to the experiments to check binocular visual acuity in photopic and mesopic conditions, contrast sensitivity and time of recovery after glare. Table 3 provides the characteristics of the panel. Participants read and signed an informed consent form; they received a financial compensation after the experiment.

\subsection{Experimental setting and protocol}

\subsubsection{Static protocol}

In order to investigate urban lighting, the experiment was based on common use conditions, rather than on a factorial plan with photometric/geometric variables (e.g.

\footnotetext{
${ }^{\mathrm{a}}$ https://www.dial.de/en/dialux/
} 
eccentricity, luminance, etc). Participants were asked to rate the discomfort glare on the de Boer scale $^{8}$ from four positions illustrated in Figure 1: $8.5 \mathrm{~m}$ (circle), $6.5 \mathrm{~m}$ (square), $4.5 \mathrm{~m}$ (triangle) and $2.5 \mathrm{~m}$ (star) ahead of the first pole (the closest to the observers). The de Boer scale as follows was translated into French:

- 1 Unbearable (Insupportable)

- 2

- 3 Disturbing (Dérangeant)

- 4

- 5 Just admissible (Supportable)

- 6

- 7 Satisfactory (Acceptable)

- 8

- 9 Unnoticeable (Négligeable)

They were asked to look at one of two targets (see Figure 1). The first target was straight ahead 14 m away from the first luminaire (Lum1 in Figure 1); the second target was also $14 \mathrm{~m}$ away from Lum 1 but $10^{\circ}$ to the left (one for each of the four positions).

In order to study the impact of the number of luminaires on discomfort glare, each lantern was judged with one (1S) or two luminaires $(2 \mathrm{~S})$ switched on. In condition $1 \mathrm{~S}$, the luminaire closest to the observer (Lum1) was switched on; in the condition 2S, both luminaires were switched on. Sixty-four ratings (4 lanterns x 4 positions x 2 gaze directions x 2 numbers of luminaires) were collected in the Static protocol for each participant.

\subsubsection{Dynamic protocol}

In the dynamic, more realistic condition, participants were asked to walk from the first static position ( $8.5 \mathrm{~m}$ from Lum 1 ) to the target ahead, in front of them (target $0^{\circ}$ in Figure 1 ), looking continuously at this target. The gaze direction was thus parallel to the road axis. Then, 
they were asked to rate on the de Boer scale the global discomfort glare they felt during the walk. Each participant made eight such ratings (4 lanterns $* 2$ numbers of luminaires).

\subsection{Experimental design, procedure and statistical analysis}

In the "Static" protocol, the four experimental factors are the lantern (A, B, C or D), the viewpoint $(8.5 \mathrm{~m}, 6.5 \mathrm{~m}, 4.5 \mathrm{~m}$ or $2.5 \mathrm{~m}$ from Lum 1$)$, the gaze direction $\left(0^{\circ}, 10^{\circ}\right.$ from the street axis) and the number of luminaires (one or two). In the "Dynamic" protocol, the two experimental factors are the lantern and the number of luminaires.

Participants came two by two. Each participant was handed a booklet where the individual order of the experimental conditions was specified. Lanterns and conditions $1 \mathrm{~S} / 2 \mathrm{~S}$ were presented in a balanced random order. For each lantern, the order of the Static and the Dynamic tasks was reversed between condition $1 \mathrm{~S}$ and $2 \mathrm{~S}$ for each participant. For the Static Protocol, based on a balanced random experimental plan, positions and directions were presented in a different order to each participant. The experimental sessions lasted about 1 hour. A short practice was carried out at the start of the session to familiarize the participants with the experimental protocol and the de Boer scale.

For data analysis, repeated measures ANOVAs ${ }^{44}$ were performed (normality was satisfied according to Kolmogorov-Smirnoff and Jarque-Béra tests). The Mauchly test was employed to test the sphericity ${ }^{45}$. The Greenhouse-Geisser correction was employed when the sphericity was not satisfied. The significance threshold was set to $\mathrm{p}=0.05$.

\subsection{Photometric description of the visual stimuli}


This section describes the photometric measurements needed to describe the stimuli and implement the glare models. Table 4 summarizes the photometric and geometric characteristics of each stimulus. All measurements and calculations were made considering an observer with eye height at $1.50 \mathrm{~m}$.

\subsubsection{Vertical illuminance}

For each stimulus, the vertical illuminance was measured at $1.50 \mathrm{~m}$ height with a LMT B520 illuminance meter aimed towards the respective target, and is reported in Table 4. In addition, Figure 2 shows the profile of vertical illuminance along the walk for the four lanterns A - D when only Lum1 is switched on.

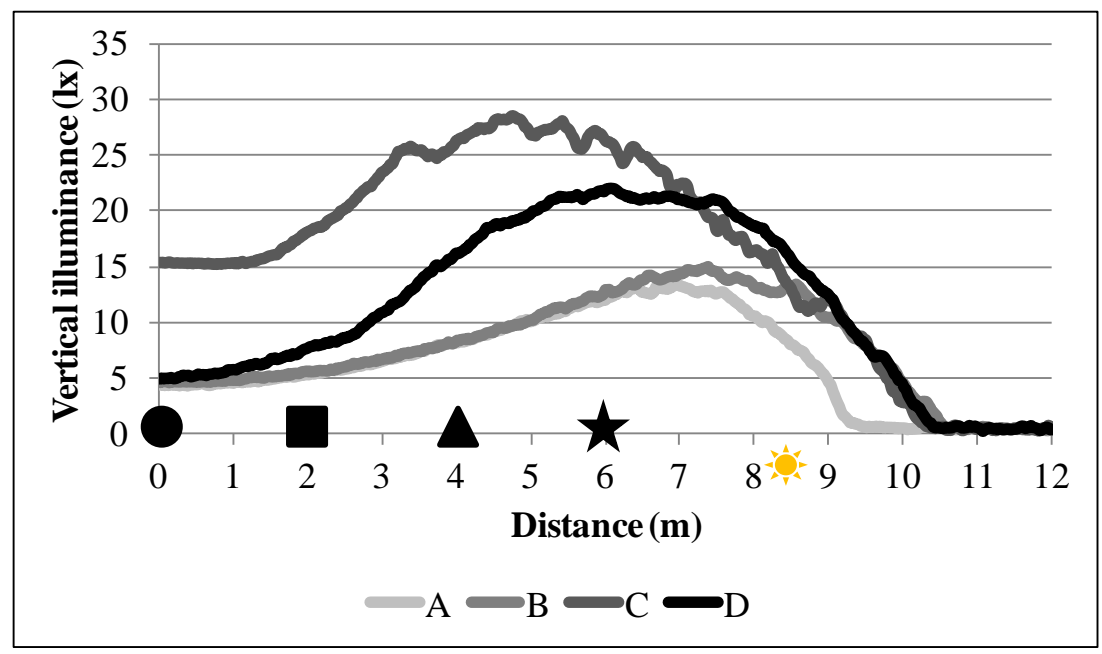

Figure 2. Vertical illuminance along the walk in condition $1 \mathrm{~S}$, for lanterns A, B, C and D. The four black symbols (circle, square, triangle \& star) correspond to the rating positions in the static protocol (see Section 2.3.1). The sun symbol marks the position of pole Lum1.

\subsubsection{Background luminance and illuminance}


Based on previous work (see Section 1.3.1), we have considered two ways of computing the background luminance from luminance maps (see Figure 3(a)). The first method uses the average luminance in the Road Zone $\left(\mathrm{L}_{\mathrm{RZ}}\right)$; the second one uses the mean luminance in a Disc Zone $30^{\circ}$ in diameter $\left(\mathrm{L}_{\mathrm{DZ}}\right)$ centred at the target which the observers were looking at (see Figure 3(a)). Thirty degree corresponds to the central visual field as defined by Bardy et $a l .{ }^{46}$ when studying central and peripheral vision in postural control while walking. A Konica Minolta CA2000A imaging photometer with a wide lens (8 mm, 1:1.4, FOV: $\left.46^{\circ} \times 46^{\circ}, 980 \times 980\right)$ captured luminance maps of the scenes (see Figure 3(a)). The average background luminance was extracted from these maps, using either $\mathrm{L}_{\mathrm{RZ}}$ or $\mathrm{L}_{\mathrm{DZ}}$.

Background illuminance values $\mathrm{E}_{\mathrm{RZ}}$ and $\mathrm{E}_{\mathrm{DZ}}$ were computed from $\mathrm{L}_{\mathrm{RZ}}$ and $\mathrm{L}_{\mathrm{DZ}}$ respectively, assuming the road and the targets were Lambertian surfaces (see Section 1.3.4).

A luminance map was also captured with all the luminaires switched off to characterize an average ambient luminance $\mathrm{L}_{\text {black }}$. The illuminance $\mathrm{E}_{\text {black }}$ was deduced from this map $\left(\mathrm{E}_{\mathrm{black}}=\pi \mathrm{L}_{\text {black }}\right)$.

\subsubsection{Source luminance and illuminance}

The source luminance was measured with a calibrated Canon EOS 70D camera (Sigma 4.5mm, 1:2.8; FOV: 180 $3648 \times 2432$ ) with fish-eye lens, connected to the Photolux software. This device makes it possible to capture a $180^{\circ}$ luminance map of the visual scene. Source areas were manually selected to compute the solid angle and mean luminance of each source. Two methods were considered for selecting the source areas: the selection encompassed either the LED module alone ( $\mathrm{L}_{\mathrm{LED}}$ in the following) or the whole lantern ( $\mathrm{L}_{\text {Lantern }}$ in the following), see Figure 3(b). 
The illuminance due to any area in the luminance map can be computed from the luminance, solid angle and eccentricity from the centre of the image (which corresponds to the gaze direction) of the pixels in the area. Vertical illuminance due to the source was thus computed, leading to two illuminance values, respectively $\mathrm{E}_{\mathrm{LED}}$ and $\mathrm{E}_{\mathrm{Lantern}}$.

(a)
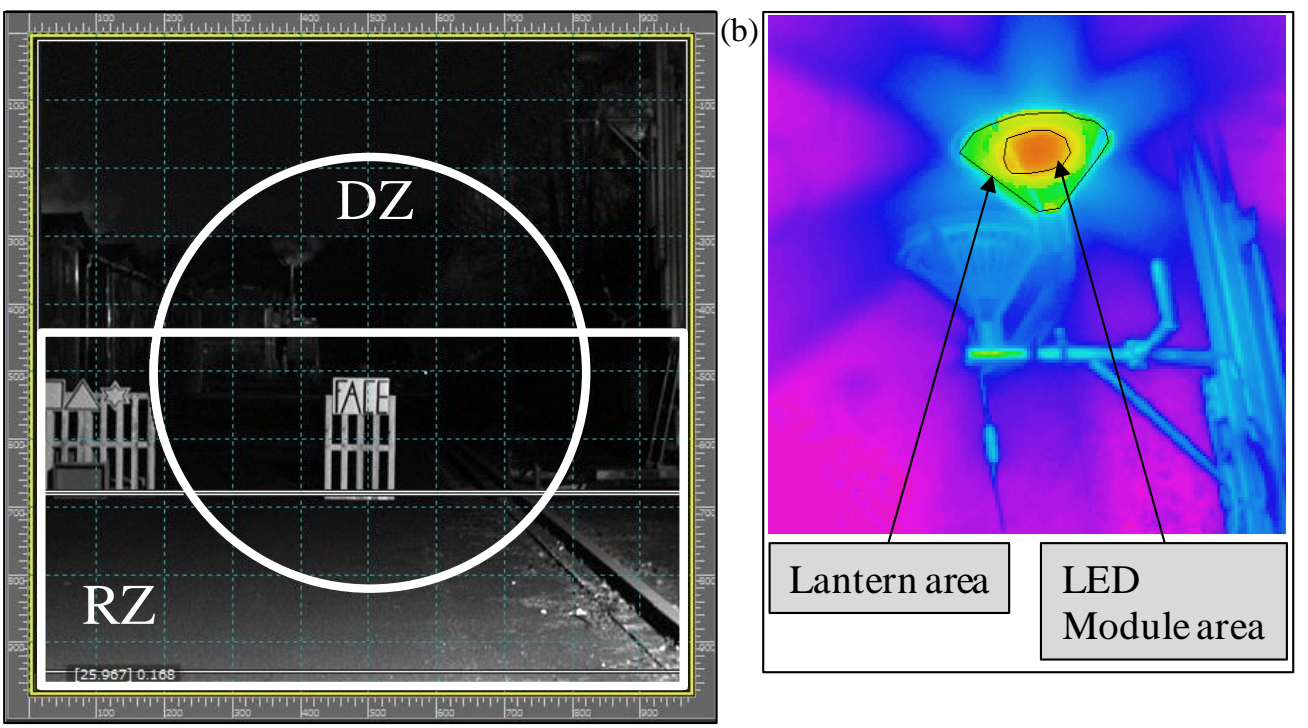

Figure 3. (a) Areas for the background luminance estimation. DZ (Disc Zone): Disc $30^{\circ}$ in diameter. RZ (Road Zone), rectangle including the road surface and the targets. (b) Areas for the source luminance estimation: LED Module area and Lantern area. 


\section{Psychovisual results}

\subsection{Preliminary analyses}

A cluster analysis was first carried out to look for potential groups of observers who share an opinion which differs from that of other groups, as well as potential outliers. Neither was found with hierarchical ascendant clustering (conducted with the percent disagreement distance and average linkage). Therefore, all data were considered in further statistical analyses.

Because of weather conditions at the time of the experiment, the road surface was wet for 20 participants and dry for 13 participants. A preliminary analysis was conducted to check if road surface condition had an effect on discomfort glare, in which case the data would possibly be split according to the road surface condition. Repeated measures ANOVA was run on the data from each protocol (Static or Dynamic), with the "Stimulus" as an intrasubject factor (64 modalities in the Static protocol, 8 modalities in the Dynamic protocol) and the "Road surface condition" as an inter-subject factor (2 modalities: dry or wet road surface). No significant effect of the road surface condition was found either in the Static protocol (Road surface condition: $\mathrm{F}(1,31)=1.20, \mathrm{p}=0.28$, Road surface condition*Stimuli: $\mathrm{F}(63,1953)=0.44, \mathrm{p}=1.00)$ or in the Dynamic protocol $(\operatorname{Road}$ surface condition: $\mathrm{F}(1,31)=$ $1.35, p=0.25$, Road surface condition*Stimuli: $F(7,217)=0.64, p=0.72)$. Therefore, road surface condition was not considered in further analyses. Only the luminance maps captured in dry conditions were considered in further computations. 


\subsection{Static protocol}

Discomfort glare ratings were collected for each type of lantern, with one or two luminaires switched on, from participants in four different positions with two gaze directions. A repeated measures ANOVA was run on the data (after Greenhouse-Geisser correction ${ }^{44}$ ). The lantern $\left(\mathrm{F}(2.45,78.39)=19.02, \mathrm{p}<0.0001, \eta^{2}=0.373\right)$, the gaze direction $(\mathrm{F}(1,32)=$ $\left.7.79, p<0.05, \eta^{2}=0.196\right)$, the number of luminaires $\left(F(1,32)=5.62, p<0.05, \eta^{2}=0.149\right)$ and the position $\left(F(2.24,71.57)=5.19 p<0.05, \eta^{2}=0.139\right)$, all had statistically significant main effects on discomfort glare. The variation of discomfort glare with the positions is significantly different depending on the lantern ("Lantern*Position": $\mathrm{F}(3.99,127.68)=16.09$, $\left.\mathrm{p}<0.0001, \eta^{2}=0.335\right)$. No other significant interaction was found (“Lantern*Direction”: $\mathrm{F}(2.92,93.37)=1.037, \mathrm{p}=0.3796 ;$ "Position*Direction": $\mathrm{F}(2.27,72.76)=0.229, \mathrm{p}=0.876$ "Lantern*Number of luminaires": $F(2.50,80.04)=0.87, p=0.460)$; "Number of luminaires*Position": $\mathrm{F}(2.28,73.00)=1.731, \mathrm{p}=0.166$; "Number of luminaires*Direction": $\mathrm{F}(1,32)=0.134, \mathrm{p}=0.717)$

Table 5 shows the mean and standard deviation values for each factor. The higher the rating, the lower the discomfort glare. Post-hoc Tukey tests ${ }^{44}$ were conducted in order to compare the lantern performance and showed no significant difference between Lanterns A and $\mathrm{B}$, which produced significantly less glare than lantern $\mathrm{D}$, which in turn produced significantly less glare than Lantern C. Lanterns A and B, with opalescent and frosted glass, produced significantly less glare than the two others, with an "acceptable" average rating across positions (see Table 5). Discomfort under Lantern D was judged on average 
“acceptable" 8.5 m from Lum1, but "just admissible" nearer (4-6 m). Finally, Lantern C which produces the highest luminance (see Table 4) was the most glaring.

According to the post-hoc Tukey tests, participants felt less comfortable when looking along the road than when looking away from the luminaires. More discomfort was experienced at $6.5 \mathrm{~m}$ and $4.5 \mathrm{~m}$ from Lum 1 than at $8.5 \mathrm{~m}$ or $2.5 \mathrm{~m}$. In addition, lighting installations were rated as significantly less disturbing when two luminaires were switched on. The situations with the most glare were at $6.5 \mathrm{~m}$ and $4.5 \mathrm{~m}$ from Lum1 looking straight ahead, with a single luminaire $\left(\mathrm{M}=5.95\left[1 \mathrm{~S}, 6.5 \mathrm{~m}, 0^{\circ}\right] ; \mathrm{M}=5.92\left[1 \mathrm{~S}, 4.5 \mathrm{~m}, 0^{\circ}\right]\right)$.

\subsection{Dynamic protocol}

A repeated measures ANOVA (after Greenhouse-Geisser correction) and post hoc Tukey tests were conducted. An effect of the Lantern was found $(F(2.51,80.21)=12.07, p<$ $\left.0.0001, \eta^{2}=0.274\right)$. There was no effect of the number of luminaires $(F(1,32)=1.30, p=$ 0.2633), however, an interaction was found $(\mathrm{F}(2.60,83.34)=3.97, \mathrm{p}=0.0103<0.05$, $\left.\eta^{2}=0.110\right)$

According to the post-hoc Tukey tests, there is no significant difference between Lanterns A and B. Lantern A produces significantly less glare than Lanterns C and D. Lanterns B and D produce significantly less glare than Lantern C. The effect of the Lantern is consistent with the results from the static protocol (see Section 3.2). However, as shown in Table 5, the de Boer rating after a walk is higher (+0.4 in average), suggesting that pedestrians feel less discomfort after walking than when standing in front of a static scene. The same levels of discomfort glare were found whatever the number of luminaires, except for the lantern $\mathrm{C}$, which produced more glare with two luminaires. 


\subsection{Photometry and discomfort glare}

The vertical illuminance at the eye-height is negatively correlated to the mean ratings on the de Boer scale $\left(\mathrm{R}^{2}=0.711\right)$. The correlation is even stronger when the data is restricted to conditions $1 \mathrm{~S}\left(\mathrm{R}^{2}=0.776\right)$ or $2 \mathrm{~S}\left(\mathrm{R}^{2}=0.796\right)$. Direct vertical illuminance from the source, computed from the whole lantern, is also correlated with glare (condition $1 \mathrm{~S}: \mathrm{R}^{2}=0.717$ ).

Various luminance indices can be computed to describe non-uniform sources: mean luminance values $\mathrm{L}_{\mathrm{LED}}$ and $\mathrm{L}_{\text {Lantern }}$ (see Section 2.5.3 and Figure 3) as well as maximum luminance $\mathrm{L}_{\max }$ (this index does not depend on the measurement area). Correlations between these luminance indices and the mean glare ratings have been computed: in Condition 1S, the correlation is stronger with $\mathrm{L}_{\mathrm{LED}}\left(\mathrm{R}^{2}=0.741\right)$ than with $\mathrm{L}_{\mathrm{Lantern}}\left(\mathrm{R}^{2}=0.457\right)$; the strongest correlation is found with $\mathrm{L}_{\max }\left(\mathrm{R}^{2}=0.771\right)$.

We also investigated the influence of photometric variations during the walk in the dynamic protocol. The differences $\Delta \mathrm{E}$ between the maximum and minimum illuminances, as well as the mean illuminance along the path (up to the first pole in condition $1 \mathrm{~S}$, up to the second pole in condition $2 \mathrm{~S}$ ) were computed. Because the source luminance was only available at the four positions of the static protocol, $\Delta \mathrm{L}$ and the mean luminance were estimated from those four values. Mean ratings in the dynamic protocol were negatively correlated to $\Delta \mathrm{L}_{\max }\left(1 \mathrm{~S}: \mathrm{R}^{2}=0.584\right)$ and to $\Delta \mathrm{L}_{\mathrm{LED}}\left(1 \mathrm{~S}: \mathrm{R}^{2}=0.512\right)$ and more weakly to $\Delta \mathrm{L}_{\text {Lantern }}\left(1 \mathrm{~S}: \mathrm{R}^{2}=0.359\right)$ and to $\Delta \mathrm{E}\left(\mathrm{R}^{2}=0.427\right)$. The mean of the vertical illuminance $\mathrm{E}_{\text {tot }}$ $\left(\mathrm{R}^{2}=0.773,1 \mathrm{~S}: \mathrm{R}^{2}=0.900,2 \mathrm{~S}: \mathrm{R}^{2}=0.976\right)$, the mean of $\mathrm{L}_{\mathrm{LED}}\left(\mathrm{R}^{2}=0.549\right)$, the mean of the maximum illuminance $\left(\mathrm{R}^{2}=0.677,1 \mathrm{~S}: \mathrm{R}^{2}=0.876,2 \mathrm{~S}: \mathrm{R}^{2}=0.946\right)$ and the mean of the maximum luminance $\left(\mathrm{R}^{2}=0.612\right)$ on the pathway, were all negatively correlated with the 
mean global ratings collected from the dynamic protocol. The strongest correlation was found with the mean of the vertical illuminance.

\section{Comparisons of discomfort glare models}

This section explores the relevance of the models developed in previous work to predict glare ratings on the de Boer scale, for pedestrian applications. It also investigates the sensitivity of these models when implemented in complex scenes (heterogeneous light source and heterogeneous background). Predictions from the models presented in Section 1.2 (Sch74, Lin14, Lin15, Bul11) are compared to mean ratings obtained from our psychovisual experiment in the static protocol (considered as the reference).

\subsection{Model implementation}

The four models have been evaluated with several variations in their input parameters. The background was taken as the road zone or as a $30^{\circ}$ circular visual field (see Section 2.5.2). Lin 14 predictions were computed with the source taken as the whole lantern or as the LED Module inside the lantern. When the source vertical illuminance was needed (Sch74, Lin15, Bul11), it was computed from the whole lantern area.

In Bullough's models ${ }^{9,12,31}$, the ambient illuminance $\mathrm{E}_{\mathrm{a}}$ is the vertical illuminance when all the luminaires are switched off $\left(E_{a}=E_{b l a c k}\right.$ in Table 4). Our understanding is that the surrounding illuminance $\mathrm{E}_{\mathrm{s}}$ quantifies the indirect illuminance due to the lighting installation. For implementation, $\mathrm{E}_{\mathrm{s}}$ was either computed following the subtracting method described in 
the original paper ${ }^{9}\left(E_{\mathrm{S}}=\mathrm{E}_{\mathrm{tot}}-\left(\mathrm{E}_{\mathrm{l}}+\mathrm{E}_{\mathrm{a}}\right)\right)$, or defined as $\mathrm{E}_{\mathrm{RZ}}$ or $\mathrm{E}_{\mathrm{DZ}}$, computed from the background luminance maps. Because the angular size of the nearest light source was always found higher than $0.3^{\circ}$, whatever the lantern, the source luminance was taken into account in model Bul11 $1^{12}$ (Equation 3). According to preliminary tests, Bul11 performs better when $\mathrm{L}_{\mathrm{s}}$ is set to $\mathrm{L}_{\text {Lantern }}$ than $\mathrm{L}_{\max }$, and that's why $\mathrm{L}_{\mathrm{s}}$ is set to $\mathrm{L}_{\text {Lantern }}$ in the following.

Lin et al. ${ }^{13}$ proposed to correct their model predictions by offset based on the correlated colour temperature of the source (but the model formulation does not take this variable into account, see Equation 4). An offset of -1.5 could apply with 4000K LEDs. However, with such an offset, the predictions were outside the de Boer scale range, and that's why we did not take it into account.

\subsection{Relevance of the glare models for pedestrian applications}

Predictions from the glare models are compared with the experimental data collected under a single luminaire in Section 4.2.1. The sensitivity of the results to the model implementation is discussed in Section 4.2.2. Finally, the number of sources is considered in Section 4.2.3.

\subsubsection{Relevance with a single source}

The GCM model cannot be implemented in our experiment because the luminous intensity at $88^{\circ}$ of the lanterns tested in the study is nil. $\mathrm{I}_{88}$ was deduced from road lighting, with higher poles than in urban lighting, for the point of view of the drivers. $88^{\circ}$ corresponds to a viewing distance of $115 \mathrm{~m}$ from a $4 \mathrm{~m}$-height luminaire, which is far beyond where pedestrians look ${ }^{37,47}$. Therefore, this model seems not to be relevant for pedestrian lighting. 


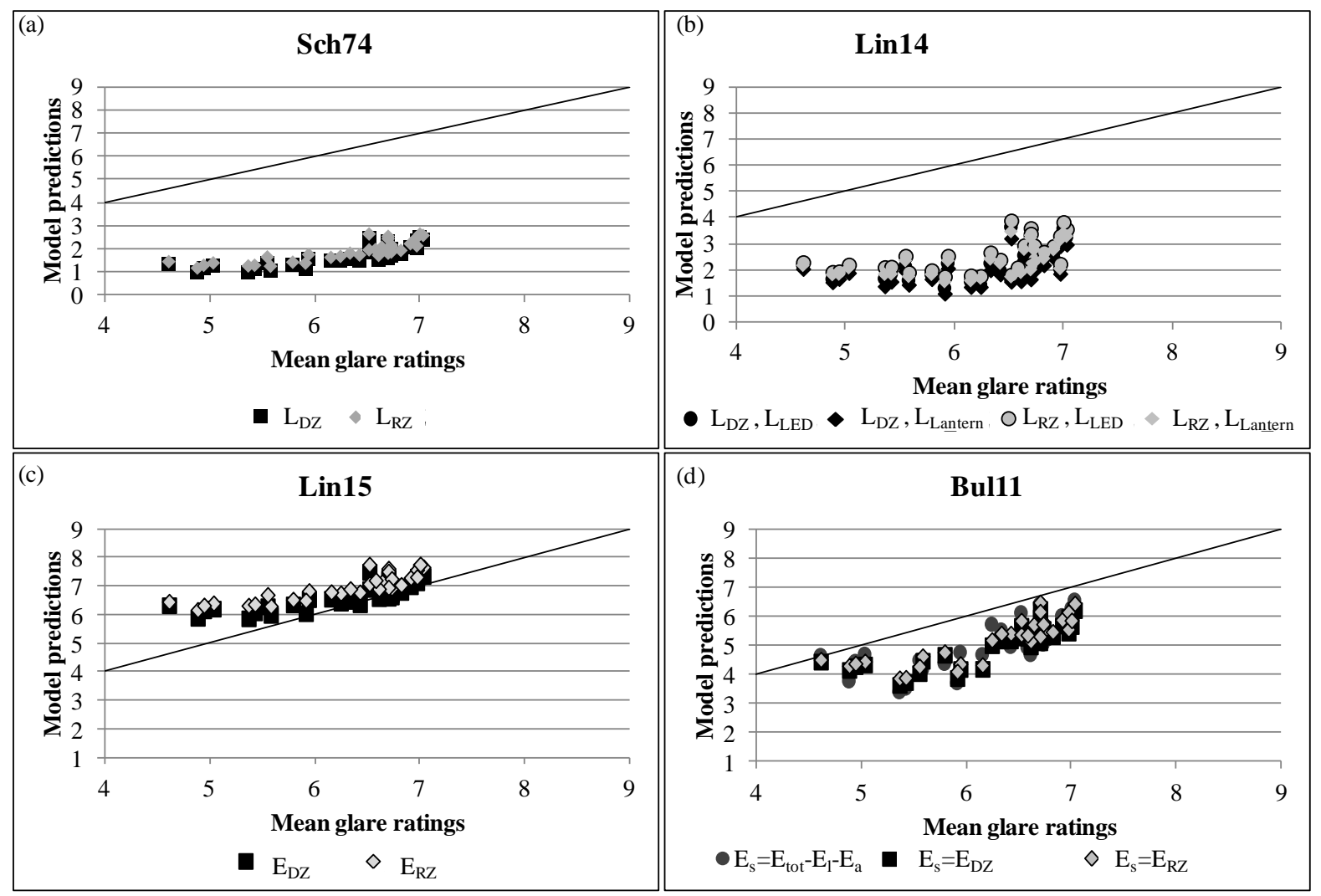

Figure 4. Model predictions versus mean glare ratings in condition 1S: (a) Sch74, (b) Lin14, (c) Lin15, (d) Bul11. For each model, the predictions vary depending on the implementation choices (e.g. $\mathrm{L}_{\mathrm{DZ}}$ vs. $\mathrm{L}_{\mathrm{RZ}}$ in $\mathrm{Sch} 74$ )

Figure 4 compares predictions from the other models with the mean glare ratings in the static protocol, for each stimulus, in condition 1S. For each model, predictions using the different computational methods are presented (e.g. $\mathrm{L}_{\mathrm{DZ}}$ and $\mathrm{L}_{\mathrm{RZ}}$ to compute the background luminance in the Sch74 model). Table 6 presents the Root Mean Square Error (RMSE), which is a global index of the errors with respect to the experimental data. The Spearman correlation $\left(\mathrm{R}^{2}\right)$ is also reported.

The Sch74 and Lin14 models obtained a RMSE above 3.75. The Bul11 and Lin15 models performed much better, with a RMSE lower than 1 in some conditions. 
Lin15 is the only model to overestimate the de Boer score. It is also the best one in terms of RMSE, with a RMSE as low as 0.60 when the background illuminance is computed from the disc zone (Table 6).

Bul11 underestimates the glare ratings, but the model performs quite well, with a RMSE of [1.1-1.3]. The lowest RMSE is obtained when $\mathrm{E}_{\mathrm{s}}$ is taken as the mean illuminance in the road zone.

As illustrated in Figure 4(a), predictions from Sch74 strongly overestimate the discomfort glare (i.e. underestimate the rating) with RMSE > 4, on a scale range of 1 to 9. This model was developed for automotive lighting applications and was based on experimental data were the source eccentricity was small (between $0.16^{\circ}$ and $1.5^{\circ}$ ), which is far from the range considered in our study $\left(9^{\circ}\right.$ to $\left.62^{\circ}\right)$. Also, the illuminances were between $10^{-3}$ and $10^{1} \mathrm{~lx}$ in Schmidt-Clausen and Bindels' experiment ${ }^{7}$. Therefore, the illuminances experienced by the pedestrians were from one to three orders of magnitude higher than those which fitted the Sch74 model. Our results suggest that Sch74 model shouldn't be generalized outside the photometric range of the original model. While the absolute level is much underestimated, Sch74 predictions are more relevant for ranking (see Table $6, \mathrm{R}^{2}=0.75$ ).

Similarly, Lin14 predictions strongly underestimate the ratings (see Figure 4(b), RMSE > 3.75). Again, it may be due to the fact that the background luminance is outside the range of the data used for model fitting (see Table 1). Moreover, correlations are low with this model (down to $\mathrm{R}^{2}=0.385$ ). 
As shown in Figure 4, for the most uncomfortable stimuli, the mean ratings vary between 4 and 6 on the de Boer scale whereas the predictions, whatever the model, are nearly constant. This is discussed in Section 5.

\subsubsection{Sensitivity of model predictions to the implementation choices}

Whatever the model (Sch74, Lin14, Lin15 or Bul11), the predictions are always higher in the de Boer scale with the RZ background than with the DZ background $(+0.2 / 0.3)$, because $\mathrm{L}_{\mathrm{RZ}}>\mathrm{L}_{\mathrm{DZ}}$. Therefore, the RMSE is lower with $\mathrm{L}_{\mathrm{RZ}}$ (or $\mathrm{E}_{\mathrm{RZ}}$ ) for models which underestimate the de Boer ratings, and higher for Lin 15 which overestimates the ratings. The RMSE between model predictions computed with RZ and DZ backgrounds was calculated and leads to errors of less than the 0.3 level on the de Boer scale (Sch74: 0.18, Lin14: 0.30, Lin15: 0.30, Bul11: 0.18).

Lin14 makes different predictions depending on the source definition (photometry and geometry). This impacts both the source luminance $\left(\mathrm{L}_{\mathrm{LED}}>\mathrm{L}_{\mathrm{Lantern}}\right)$ and the source solid angle $\left(\mathrm{W}_{\mathrm{LED}}<\mathrm{W}_{\text {Lantern }}\right)$ but in opposite ways, so that the result is not predictable. When comparing the Lin14 predictions computed with $\mathrm{L}_{\mathrm{LED}}$ and $\mathrm{L}_{\text {Lantern, }}$ the RMSE is 0.21 on the de Boer scale.

Considering all these implementation issues and Figure 4, it is clear that the glare predictions are more dependent on the models than on their implementation.

\subsubsection{Relevance of the glare models for two sources}

The Sch74 model is the only one which provides a way to compute discomfort glare with several sources in the visual field. There is no consensus in previous work about the effect of multiple sources on discomfort glare (see Section 1.1). Moreover, published models 
do not address the number of sources in the same way. De Boer and Schreuder ${ }^{8}$ found an improvement of +0.5 on the de Boer scale when the number of visible light sources is halved, which account for the number of luminaires per km in the GCM model. Schmidt-Clausen and Bindels ${ }^{6}$, who developed an automotive lighting model with up to five sources at low eccentricity, assume additivity in the logarithm (see Equation 6): each source illuminance and eccentricity is considered separately with the same background. Additivity is also assumed in discomfort glare models for indoor lighting, such as UGR and $\mathrm{DGI}^{23}$. For outdoor lighting, the Bullough et al.'s model predicts the discomfort glare from one source. The authors also show that a second source doesn’t impact the glare if it is at least $9^{\circ}$ away from the line of sight; it only impacts the surrounding illuminance. Lin et al. ${ }^{13,14}$ did not address the multisource issue at all.

In the following, we have considered two computational methods to estimate the discomfort glare ratings in the presence of two glare sources:

- Additive method: the discomfort glare from the two sources Lum1 and Lum2 is taken into account, with the background of condition 2S. As proposed by Schmidt Clausen and Bindels ${ }^{7}$, the contributions of both sources were added. To that purpose, we have adapted Bu108 ${ }^{\mathrm{b}}$, Lin14 and Lin15 as presented in Equation 6. These formulas can easily be generalized to $\mathrm{N}$ sources.

\footnotetext{
${ }^{\mathrm{b}}$ Bul08 instead of Bul11 was computed for Bullough's model to avoid adding bias with the additional luminance term
} 


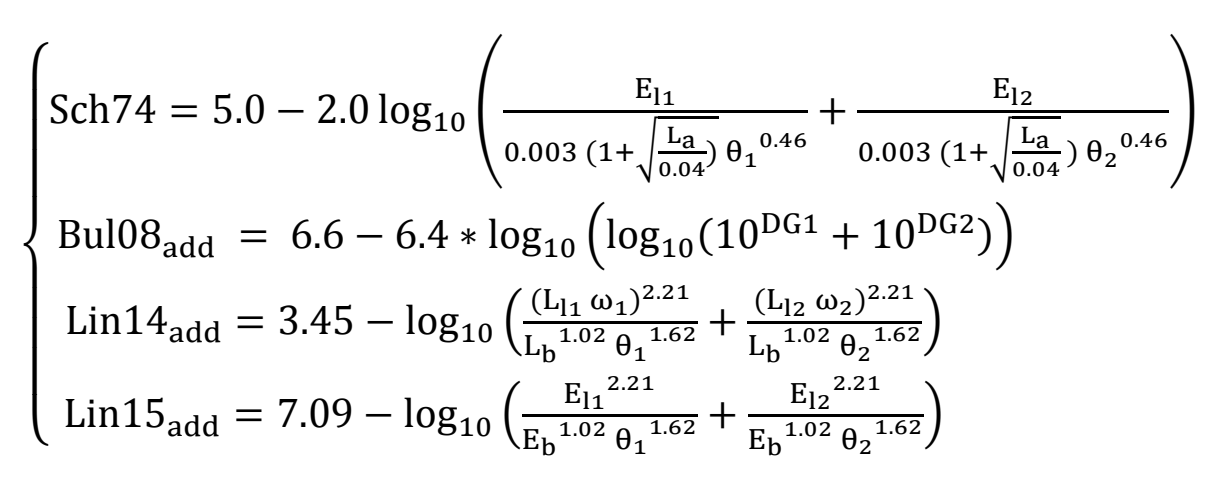

- Simplified method: discomfort glare from the closest luminaire (Lum1) alone is taken into account, under the background of condition 2 S. Lum2 only contributes to the background.

The predictions of the additive method and of the simplified method were compared. The difference always ranges between -0.1 and 0 (from -0.085 to -0.015 for $S c h 74$, from -0.02 to 0.003 for Bul08, from -0.006 to 0 for Lin15, and from -0.002 to 0 for Lin14). Thus, the contribution of Lum2 as a glare source in our experimental setting is negligible: the second luminaire is "far enough away". The contribution of Lum2 is to increase the adaptation level and therefore to decrease the discomfort glare. Such findings agree with Bullough et al. ${ }^{9}$ for urban lighting. Equation 6 allows quantifying the cumulative effect of additional glare source with the models, to see whether the additional sources have a significant effect on glare or if they are "far enough away" to be neglected.

Taking into account Lum1 alone as a glare source while increasing the background luminance due to Lum2 results in higher glare rating predictions, which is consistent with the experimental findings (see Section 3.2). The RMSE and $\mathrm{R}^{2}$ between model predictions and mean glare ratings were also computed, see Table 7 . They have the same order of magnitude 
as those obtained in condition 1S (Table 6), and thus the models have similar performances with one and two poles.

\section{Discussion}

\subsection{Findings on pedestrian discomfort glare}

A psychovisual experiment was conducted to collect judgements on pedestrian discomfort glare under various urban lighting installations in static and dynamic conditions. Lanterns with opalescent (Lantern A) and frosted glass (Lantern B) optics obtained the highest scores (i.e. produced the less glare) in our experiment. Moreover, with only one luminaire switched on, the vertical illuminance $\left(\mathrm{R}^{2}=0.776\right)$ and the maximum source luminance $\left(\mathrm{R}^{2}=0.771\right)$ were the photometric characteristics which showed the strongest correlation with the subjective data in the static protocol. Together, these results confirm previous findings ${ }^{5,30}$ which recommend frosted or opalescent optics for LED luminaires to limit maximum luminance and thereby reduce pedestrian discomfort glare.

Discomfort glare was rated as lower when assessed globally after a walk, compared to static ratings. In this case, the strongest correlation of the global ratings is obtained with the mean vertical illuminance along the path $\left(R^{2}=0.773 ; 1 S: R^{2}=0.900 ; 2 S: R^{2}=0.980\right)$. A possible interpretation would be that the discomfort glare reported after a walk is based on an average feeling. 
In urban lighting, a second luminaire may produce glare, but also contributes to an increase of the adaptation level. With the static protocol, we found that glare was significantly lower with two luminaires than with a single luminaire. With the dynamic protocol, however, we found that global ratings did not significantly differ between one or two luminaires; the second part of the walk may have affected the overall judgment more than the first part. In terms of visual adaptation, the second part of the walk with two luminaires was close to condition $1 \mathrm{~S}$ in the static protocol. The second luminaire was found to impact the background, but did not contribute much to the direct glare. This result is consistent with Bullough et al. ${ }^{9}$ and with previous pedestrian studies ${ }^{5,11}$, which found that pedestrians feel the glare from one luminaire at a time $e^{5}$, the closest one ${ }^{11}$. However, de Boer and Schreuder ${ }^{8}$ found less discomfort glare when halving the number of luminaires, so that further investigations are needed with a larger number of light sources.

As a conclusion about the protocol, the assessment of discomfort glare due to urban lighting with a static protocol (which is usually employed) and one luminaire tends to overestimate the discomfort glare for a pedestrian walking in the street. It is some kind of a "worst case" as far as discomfort glare is concerned.

\subsection{Predictions of pedestrian discomfort glare}

\subsubsection{Performance of the models}

Existing discomfort glare models have been investigated, and their predictions were compared with mean ratings considered as a reference. The main goal was to check whether these models could be applied in a real-world situation. Three models were found to overestimate the discomfort from glare, while the Lin15 model underestimates it. With RMSE 
higher than 3.7 on a scale with 9 levels, Sch74 and Lin14 models are not able to predict the mean glare ratings of our experiment, which suggests that these models should not be generalized too far away from their original data range. The illuminances employed to fit the $\operatorname{Sch} 74^{7}$ model were between $10^{-3}$ and $10^{-1} \mathrm{~lx}$, much lower than the levels expected in pedestrian lighting; the background luminance of the case study is one order of magnitude lower than the range employed to develop the model Lin14 ${ }^{13}$. Bul11 and Lin15 perform better with RMSEs lower than 1.4 and 0.9 , respectively, and could be reasonably employed to predict pedestrian glare ratings. Thus, the two models based on illuminance only performed better than the two others. This is consistent with the strong correlation between the mean ratings and the mean vertical illuminance $\left(R^{2}=0.711\right)$.

In his review of Bullough et al's paper ${ }^{9}$, Boyce highlights that the Bul11 model is restricted to zero eccentricity, and suggests that the relevance of such a model for street lighting applications, where drivers/pedestrians do not usually look straight at the sources, remains unknown. On the other hand, Bullough et al ${ }^{20}$ found that discomfort glare assessments collected in a fixed gaze experiment were highly correlated with those collected in a free gaze experiment. The low RMSE (less than 1.4) found on the de Boer scale suggests that Bul11 could be generalized to other eccentricities.

However, none of the models succeeded in predicting the discomfort glare variations among those conditions which produced the strongest glare. As these conditions (Lantern C: from $8.5 \mathrm{~m}$ to $4.5 \mathrm{~m}$; Lantern $\mathrm{D}$ : from $6.5 \mathrm{~m}$ to $2.5 \mathrm{~m}$ ) also provided the highest source luminance values (see Table 4), the poor performance may result from the underestimation of the source luminance due to measurement limitations. Therefore, efforts should be made to improve the measurement of high luminances and contrast before criticizing the models. 


\subsubsection{Direct and indirect lighting}

Bullough et al. ${ }^{9}$ made a distinction between surrounding illuminance and ambient illuminance, which are both indirect as opposed to direct illuminance from a glare source. The amount of light at the observer's eye can be split into two components: the direct one is due to the light sources and the indirect one to reflections on the surfaces in the scene. Glare happens when the direct component is much higher than the indirect component. We have investigated the same model formulation (Equation 3) with only two terms: the direct component $\mathrm{E}_{\mathrm{direc}}=\mathrm{E}_{1}$, and the indirect component $\mathrm{E}_{\text {indirect }}=\mathrm{E}_{\mathrm{s}}=\mathrm{E}_{\mathrm{a}}$ defined as the illuminance of the background when the luminaires are switched on, which is supposed to describe the background adaptation level (Dir/Ind in the following). Table 8 shows the RMSE and Spearman correlations of those predictions and the mean glare ratings. This model performs slightly better than Bull11 (see Tables 5 and 6) although the Spearman correlation $\mathrm{R}^{2}$ is lower.

\subsubsection{Implementation of the models}

The models using the source luminance as input require arbitrary assumptions about the spatial definition of the sources, which is a current issue for LED lighting because of their strong non-uniformity ${ }^{38-40,48}$. We have considered average luminance over the whole lantern and over the LED module only, which led to a small difference $(\mathrm{RMSE}=0.21$ on de Boer scale) with Lin14 predictions. But for pedestrian applications, according to Section 3.4 and previous work ${ }^{5,30}$, one of the best correlated photometric descriptions of the source is the maximum luminance. One may wonder whether the average luminance is the most suitable variable to characterize discomfort glare from of a non-uniform luminaire. Kohko et al. ${ }^{30}$ compute an "efficient luminance", first proposed by Tashiro et al. ${ }^{40}$, which is a weighted sum 
of the spatial distribution of the source luminance, and to use it as input for the source luminance in the relevant glare models. Future work is needed in order to investigate the relevance of this index for glare prediction.

Two background zones were tested in the models' implementation. The RMSEs between model predictions obtained with either method were lower than 0.30 on the ninepoint de Boer scale. Therefore, the adaptation zone does not need to be precisely defined if the luminances have the same order of magnitude over the whole background. However, our road zone was large compared to the one tested in other work ${ }^{35}$ and the influence of the adaptation region definition deserves a complete paper itself, as seen in Section 1.3.1, especially as the region definition is difficult because of the free pedestrian gaze ${ }^{37}$.

In the design stage, surrounding light elements and surfaces are generally not known and hypotheses are required (e.g. about the material, pavement etc). Glare models can be employed in a worst-case design based on road surface luminance or illuminances recommended by the standard. However, in urban areas, additional ambient lighting provided by billboards and shop windows may increase the background luminance (they may also be additional glare sources) and therefore impact the discomfort glare level predicted in the design stage.

The models based on illuminance perform better and seem easier to implement. But in urban lighting applications, both the source (direct illuminance) and the adaptation (indirect illuminance) terms need to be measured when the source is switched on. From a practical point of view, it is difficult to split the illuminance into a source component and an ambient 
component. This is, however, possible with complementary luminance measurements, or the use of a baffle at the illuminance meter ${ }^{31}$.

\subsubsection{Measurement issues}

As highlighted above, one of the main issues in using discomfort glare models is related to measurement limitations. Whatever the measurement method, some bias appears. An ILMD may be employed (as was done in this paper) to generate luminance maps and compute the average luminance over various regions (adaptation regions, light sources) as well as direct and indirect illuminances. But, the main problem with ILMDs is the underestimation of the maximum luminance. This limit may explain why, in our data, the model's predictions were nearly constant for those stimuli with the highest glare level. We agree with Slominski" that it is "very difficult if not impossible" with current ILMD devices to "precisely define the maximum luminance measured from a long distance (20-90m)". Measurement errors increase with the distance because the LED chip is included in too few photosensors, even sub-pixel; thus, the pixel luminance averages the luminance of the LED chip and of its surroundings. Measurements also depend on the lens, sensor size and number of pixels. Slominski ${ }^{49}$ recommends "increasing the focal length value or decreasing the physical size of the area recorded by a single pixel". According to him, "the best solution is to design an HDR device with lens whose field of view of a single pixel of the $B / W$ structure is precisely connected to the resolution of the human eye". A luminance meter may have a wider luminance range, but the measurement process faces the same problems for choosing the relevant measurement area; moreover, the viewfinder towards the source is manually set and the angular aperture needs to be adjusted depending on the distance to the source. Therefore, 
the measurement region is not fully controlled. On the other hand, illuminance measurement does not meet these problems. However, it is difficult to split the illuminance into a source component and an ambient component. Bullough et al. ${ }^{9}$ and Brons et al. ${ }^{31}$ proposed using a baffle in the illuminance meter to hide the source from the sensor. With such a trick, it seems difficult however to collect reproducible data.

One may speculate that computations based on the background luminance and the overall illuminance may lead to the lowest measurement uncertainty: it would first need to measure the overall illuminance, then to capture a luminance map with an ILMD. The background photometry would then be computed from the luminance map (luminance and then illuminance, based on a Lambertian reflection hypothesis). Finally, the source-related illuminance (and the source luminance) would be computed by subtracting the background illuminance from the overall illuminance. However, this is very speculative and would deserve a complete study.

\subsubsection{Conclusions on our model investigations}

Even though our results depend on the measurement and calculation choices and uncertainties, our findings do not. Two of the tested models (Bul11 and Lin15) give fair results to predict the discomfort glare on the de Boer scale in urban pedestrian settings. A simplified model based on Bul11 also leads to good results (Dir/Ind). The main issue to predict pedestrian discomfort glare is the choice of the model; predictions are not very sensitive to implementation choices.

\subsection{Limits and future work}


Our experiment was designed in an outdoor but controlled luminous environment, which would obviously be different in real-world situations (because of vehicles' headlamps, shop windows, etc.). Pilot studies would be needed to investigate the potential bias between glare ratings in controlled and field environments. Our "walking condition" is a step in this direction.

According to Miller et al. ${ }^{4,5}$, the pedestrian could feel "overhead glare" ${ }^{\text {,50 }}$, i.e. discomfort glare under the luminaires. Eccentricities above $62^{\circ}$ were not assessed in this work in the static protocol and it would be interesting in future work to quantify this overhead glare and study if the glare models are able to take it into account.

This paper focused on discomfort glare models using the de Boer scale, but other models could be tested, either developed for outdoor ${ }^{51}$ or indoor ${ }^{23,52}$ applications. Especially, Kohko et al. ${ }^{30}$ investigated the pedestrian glare under seven light sources focusing on the luminance uniformity issue. They compared the subjective data collected on the de Boer scale with indoor glare models (e.g. DGI, UGR) and recommended to use the mDGI index (DGI index where the source luminance is replaced by the efficient luminance) for both uniform and non-uniform light sources.

Finally, the dynamic protocol was a first step to include temporal variations in discomfort glare assessment. All current models were fitted to ratings in static experimental conditions. In future work, we plan to test static models in scenarios where temporal changes occur, due to body and gaze motion.

\section{General conclusion}


This work investigates the discomfort glare experienced by pedestrians under various urban lighting installations. Psychovisual tests were conducted on a closed outdoor area with four urban lighting installations to collect discomfort glare judgments. The results confirm previous work ${ }^{5,30}$ which recommended optics to limit maximum luminance and vertical illuminance. Four predictive models have been tested with our data. The models from Lin $e t$ al. $(2015)^{14}$ and Bullough et al. $(2008,2011)^{9,12}$ provided the best predictions of the experimental data. Measurement and calculation choices were needed in order to implement these models in complex visual scenes. Little impact of the background and source definition was found on the model predictions. However, measurement uncertainties (e.g. underestimation of the source luminance) may impact the accuracy of the models, especially for high glare levels (i.e. low ratings). Such limitations deserve to be investigated in future work to ensure an accurate implementation of discomfort glare models in urban environments. In addition, current models do not take into account the temporal changes in the pedestrian's glare experience. Therefore, in future work, we plan to investigate the glare rating during a walk.

\section{Funding}

This work was supported by Thorn Lighting.

\section{Acknowledgments}


The authors would like to thank Manuel Le Pape for his help, Alexandre Taron for his availability, his help and the facilities, Eric Dumont for his contributions and helpful advice, and all the participants who took part in the experiment.

\section{References}

1. Raynham P. An examination of the fundamentals of road lighting for pedestrians and drivers. Lighting Research and Technology 2004; 36(4): 307-316.

2. Kostic A, Djokic L. Subjective impressions under LED and metal halide lighting. Lighting Research and Technology 2014; 46(3): 293-307.

3. Luo W, Puolakka M, Zhang Q, Yang C, Halonen L. Pedestrian way lighting: user preferences and eye fixation measurements. Ingeniria Illuminatului 2013; 15(1): 1934.

4. Miller NJ, Koltai RN, McGowan TK. Pedestrian Friendly Outdoor Lighting. Gateway Report PNNL-23085, Portland, OR: Pacific Northwest National Laboratory, 2015.

5. Miller NJ, McGowan TK. Correspondence: Glare in pedestrian-friendly outdoor lighting. Lighting Research and Technology 2015; 47: 760-762.

6. Behar-Cohen F, Martinsons C, Vienot F, Zissis G, Barlier-Salso A, Cesarini JP, Enouf O, Garcia M, Picaud S, Attia D. Light-emitting diodes (LED) for domestic lighting: Any risks for the eye? Progress in Retinal and Eye Research 2011; 30(4): 239-257.

7. Schmidt-Clausen H, Bindels J. Assessment of discomfort glare in motor vehicle lighting. Lighting Research and Technology 1974; 6(2): 79-88.

8. de Boer JB, Schreuder DA. Glare as a criterion for quality in street lighting. Transactions of the Illuminating Engineering Society 1967; 32(2): 117-135.

9. Bullough JD, Brons JA, Qi R, Rea MS. Predicting discomfort glare from outdoor lighting installations. Lighting Research and Technology 2008; 40(3): 225-242. 
10. Zhu X, Demirdes H, Gong X, Lai J, Heynderickx I. The luminaire beam-shape influence on discomfort glare for led road lighting. In: Proceedings on MidTerm CIE 2013, 15-16 April 2013, Paris, 2013: pp. 331-339.

11. Viliunas V, Vaitkevicius H, Stanikunas R, Vitta P, Bliumas R, Auskalnyte A, Tuzikas A, Petrulis A, Dabasinskas L, Zukauskas A. Subjective evaluation of luminance distribution for intelligent outdoor lighting. Lighting Research and Technology 2014; 46(4): 421-433.

12. Bullough JD, Sweater-Hickocox K, Narendran N. A method for estimating discomfort glare from exterior lighting system. Alliance for Solid-State Illumination Systems and Technologies 2011; 9: 1-7.

13. Lin Y, Liu Y, Sun Y, Zhu X, Lai J, Heynderickx I. Model predicting discomfort glare caused by LED road lights. Optics Express 2014; 22(15): 18056-18071.

14. Lin Y, Fotios S, Wei M, Liu Y, Guo W, Sun Y. Eye movement and pupil size constriction under discomfort glare. Investigative Ophthalmology and Visual Science 2015; 56(3): 1649-1656.

15. Mayeur A, Bremond R, Bastien C. Effect of task and eccentricity of the target on detection thresholds in mesopic vision: implications for road lighting. Human Factors 2008; 50(4): 712-721.

16. Sivak M, Flannagan M, Ensing M, Simmons CJ. Discomfort Glare is Task Dependent. Report No. UMTRI-89-27. Ann Arbor, MI: University of Michigan Transportation Research Institute, 1989.

17. Land MF, Lee DN. Where do we look when we steer. Nature 1994; 369(6483): 742744.

18. Lappi O. Future path and tangent point models in the visual control of locomotion in curve driving. Journal of Vision 2014; 14(12): 1-22.

19. Tom A, Granie MA. Gender differences in pedestrian rule compliance and visual search at signalized and unsignalized crossroads. Accident Analysis and Prevention 2011; 43: 1794-1801.

20. Bullough J, Van Derlofske J, Fay C, Dee P. Discomfort glare from headlamps: Interactions among spectrum, control of gaze and background light level. SAE 
Technical Paper 2003-01-0296. Warrendale, PA: Society of Automotive Engineers, 2003.

21. Hickcox KS, Narendran N, Bullough JD, Freyssinier JP. Effect of different coloured luminous surrounds on LED discomfort glare perception. Lighting Research and Technology 2013; 45(4): 464-475.

22. Bullough JD, Fu Fu Z, Derlofske JV. Discomfort and disability glare from halogen and HID headlamp systems. Advanced Lighting Technology for Vehicles 2002: 1-5, SP-1668.

23. Clear RD. Discomfort glare: What do actually know? Lighting Research and Technology 2013; 45(2): 141-158.

24. Niedling M, Kierdorf D, Völker S. Influence of a glare sources spectrum on discomfort and disability glare under mesopic conditions. In: Proceedings on MidTerm CIE 2013, 15-16 April, 2013, Paris, France, 2013: pp. 340-347.

25. Sivak M, Flannagan MJ, Traube EC, Kojima S. The influence of stimulus duration on discomfort glare for persons with and without visual correction. Transportation Human Factors 1999; 1(2): 147-158.

26. Theeuwes J, Alferdinck JWAM, Perel M. Relation between glare and driving performance. Human Factors 2002; 44(1): 95-107.

27. Gibbons RB, Edwards C. A review of disability and discomfort glare research and future direction. $18^{\text {th }}$ TRB Biennial Visibility Symposium, College Station TX, USA, 17-19 April 2007.

28. Commission Internationale de 1'Eclairage. Glare and Uniformity in Road Lighting Installations, CIE Publication CIE-031-1976. Vienna: CIE, 1976

29. Sammarco JJ, Mayton AG, Lutz T, Gallagher S. Discomfort glare comparison for various LED cap lamps. IEEE Transactions on Industry Applications 2010; 47(3): 1168-1174.

30. Kohko S, Ayama M, Iwata M, Kyoto N, Toyota T. Study on evaluation of LED lighting glare in pedestrian zones. Journal of Light and Visual Environment 2015; doi: 10.2150/jlve.IEIJ150000566 
31. Brons JA, Bullough JD, Rea MS. Outdoor site-lighting performance: a comprehensive and quantitative framework for assessing light pollution. Lighting Research and Technology 2008; 40(3): 201-224.

32. Van Bommel W. Road Lighting, Fundamentals, Technology and Application, London: Springer, 2015.

33. Cengiz C, Kotkanen H, Puolakka M, Lappi O, Lehtonen E, Halonen L, Summala H. Combined eye-tracking and luminance measurements while driving on a rural road: Towards determining mesopic adaptation luminance. Lighting Research and Technology 2014; 46(6): 676-694.

34. Davoudian N, Raynham P. What do pedestrians look at night? Lighting Research and Technology 2012; 44(4): 438-448.

35. Uchida T, Ayama M, Akashi Y, Hara N, Kitano T, Kodaira Y, Sakai K. Adaptation luminance simulation for CIE mesopic photometry system implementation. Lighting Research and Technology 2016; 48(1): 14-25.

36. Maksimainen M, Puolakka M, Tetri E, Halonen L. Veiling luminance and visual adaptation field in mesopic photometry. Lighting Research and Technology 2016. First published 22 March 2016, doi: 10.1177/1477153516637400.

37. Foulsham T, Walker E, Kingstone A. The where, what and when of gaze allocation in the lab and the natural environment. Vision Research 2011; 51(17): 1920-1931.

38. Ayama M, Tashiroi T, Kawanobe S, Kimura-Minoda T, Kohko S, Ishikawa T. Discomfort glare of white led sources of different spatial arrangements. In: Proceedings on MidTerm CIE 2013, 15-16 April 2013, Paris, 2013: pp.119-122.

39. Higashi H, Koga S, Kotani T. The development of evaluation for discomfort glare in led lighting of indoor work place: the effect of the luminance distribution of luminous parts on subjective evaluation. In: Proceedings on MidTerm CIE 2013, 15-16 April 2013, Paris, 2013: pp. 648-656.

40. Tashiro T, Kawanobe S, Kimura-Minoda T, Kohko S, Ishikawa T, Ayama M. Discomfort glare for white LED light sources with different spatial arrangements. Lighting Research and Technology 2014; 47(3): 316-337. 
41. de Boer JB. Lighting of traffic routes. Philips Technical Review 1961-62; 23: 258-272.

42. Commission Internationale de l'Eclairage. Road Surfaces and Lighting. CIE Publication 066:1984, Vienna: CIE, 1984.

43. European Committee for Standardisation (CEN). Road Lighting - Part 2: Performance Requirements. European Standard EN 13201-2, Brussels: CEN, 2003.

44. Howell DC. Statistical Methods for Psychology, 6th edition Belmont, CA: Duxbury Press, 2008.

45. Mauchly JW. Significance test for sphericity of a normal n-variate distribution. The Annals of Mathematical Statistics 1940; 11 (2): 204-209.

46. Bardy B, Warren WH, Kay BA. The role of central and peripheral vision in postural control during walking. Perception and Psychophysics 1999; 61(7): 1356-1368.

47. Fotios S, Uttley J, Cheal C, Hara N. Using eye-tracking to identify pedestrians' critical visual tasks, Part 1. Dual task approach. Lighting Research and Technology 2015; 47(2): 133-148.

48. Kasahara T, Aizawa D, Irikura T, Moriyama T, Toda M, Iwamoto M. Discomfort glare caused by white LED light sources. Journal of Light and Visual Environment 2006: 30(2): 95-103.

49. Slominski S, Identifying problems with luminaire luminance measurements for discomfort glare analysis. Lighting Research and Technology 2016; 48(5): 573-588.

50. Boyce PR, Hunter CM, Inclan C. Overhead glare and visual discomfort. Journal of the Illuminating Engineering Society 2003; 32(1): 73-88.

51. Bennett CA. Characterizing discomfort glare from roadway lighting. Transaction Research Record 1149. In: Visibility for highway guidance and hazard detection, 1987.

52. Luckiesh M. Guth SK. Brightnesses in visual field at borderline between comfort and discomfort (BCD). Illuminating Engineering 1949; 44: 650-670. 


\section{Figure captions}

Figure 1. Experimental setup with both luminaires switched on (left) and illustration of the experimental settings (right). The geometrical shapes (circle, square, triangle, star) correspond to the viewing positions in the "Static" protocol; targets $0^{\circ}$ and $10^{\circ}$ are used to control the participants' gaze direction.

Figure 2. Vertical illuminance along the walk in condition 1S, for lanterns A, B, C and D. The four black symbols (circle, square, triangle \& star) correspond to the rating positions in the static protocol (see Section 2.3.1). The sun symbol marks the position of pole Lum1.

Figure 3. (a) Areas for the background luminance estimation. DZ (Disc Zone): Disc $30^{\circ}$ in diameter. RZ (Road Zone), rectangle including the road surface and the targets. (b) Areas for the source luminance estimation: LED Module area and Lantern area. 
Figure 4. Model predictions versus mean glare ratings in condition 1S: (a) Sch74, (b) Lin14, (c) Lin15, (d) Bul11. For each model, the predictions vary depending on the implementation choices (e.g. $\mathrm{L}_{\mathrm{DZ}}$ vs. $\mathrm{L}_{\mathrm{RZ}}$ in $\mathrm{Sch} 74$ ) 
Table 1. Range of values for variables used for modelling discomfort glare in previous work ${ }^{7-}$ 9,12-14. The last column shows the experimental data used in this paper.

\begin{tabular}{|c|c|c|c|c|c|}
\hline $\begin{array}{l}\text { Geometric } \\
\text { and } \\
\text { photometric } \\
\text { variables }\end{array}$ & $\begin{array}{l}\text { GCM } \\
\text { Glare } \quad \text { Control } \\
\text { Mark (data from } \\
\text { De Boer and } \\
\text { Schreuder, } 1967)^{8}\end{array}$ & $\begin{array}{ll}\text { Sch74 } & \\
\text { (Schmidt } & \text { Clausen } \\
\text { and } & \text { Bindels, } \\
1974)^{7} & \end{array}$ & $\begin{array}{l}\text { Lin14 } \\
(\operatorname{Lin} \\
2014)^{13}\end{array}$ et al. & $\begin{array}{l}\text { Lin15 } \\
(\text { Lin et al., 2015) }\end{array}$ & $\begin{array}{l}\text { Bul08/Bul11 } \\
\text { (Bullough et al., } \\
\text { Bullough et al., 2011) }\end{array}$ \\
\hline $\begin{array}{l}\text { Source } \\
\text { eccentricity }\end{array}$ & $\begin{array}{l}6 \text { or } 11 \text { poles; in } \\
\text { scale } 1: 50\end{array}$ & $0.16-1.5^{\circ}$ & $10,15,20^{\circ}$ & $2,4,8,16^{\circ}$ & n.a. \\
\hline $\begin{array}{l}\text { Source } \\
\text { photometry }\end{array}$ & $\begin{array}{l}\text { I80 : } \quad 100 \mathrm{~cd}- \\
5000 \mathrm{~cd} \text { (5 stages) }\end{array}$ & $10^{-3}-10^{-1} 1 \mathrm{x}$ & $10^{4} \mathrm{~cd} / \mathrm{m}^{2}$ & $10^{1}-10^{2} 1 x(20-3001 x)$ & $\begin{array}{l}10^{1}-10^{2} 1 \mathrm{x} \\
10^{4}-10^{5} \mathrm{~cd} / \mathrm{m}^{2} \\
\mathrm{E}_{\mathrm{s}}=10^{-2} 1 \mathrm{x}\end{array}$ \\
\hline $\begin{array}{l}\text { Background } \\
\text { photometry }\end{array}$ & $\begin{array}{l}0.34-6.7 \mathrm{~cd} / \mathrm{m}^{2}(4 \\
\text { stages })\end{array}$ & $10^{-3}-10^{-1} \mathrm{~cd} / \mathrm{m}^{2}$ & $10^{0}-10^{1} \mathrm{~cd} / \mathrm{m}^{2}$ & $0,10,200 \mathrm{~lx}$ & $\begin{array}{l}(0.01-1.61 x) \\
10^{-2}-10^{0} 1 \mathrm{x}\end{array}$ \\
\hline Source size & $\begin{array}{l}F: 0.007,0.07 \text { or } \\
0.25 \mathrm{~m}^{2}\end{array}$ & n.a. & $10^{-5} \mathrm{sr}$ & n.a. (distance $1.5 \mathrm{~m})$ & n.a. (distance $3-20 \mathrm{~m}$ ) \\
\hline
\end{tabular}


Table 2. Lighting characteristics. Average illuminances were measured on a $13.3 \mathrm{~m}$ x $5.5 \mathrm{~m}$ grid, according to the NF-EN13201 ${ }^{43}$ standard. In condition $2 \mathrm{~S}$ (two luminaires), Lum1 and Lum2 were located on the left corners of the rectangular grid ${ }^{43}$. In condition $1 \mathrm{~S}$ (one luminaire), Lum1 was located in the middle of the left side of the rectangular grid.

\begin{tabular}{|c|c|c|c|c|}
\hline Lantern & $\mathrm{A}$ & $\mathrm{B}$ & $\mathrm{C}$ & $\mathrm{D}$ \\
\hline Optics & $\begin{array}{l}\text { Cover opalescent } \\
\text { glass }\end{array}$ & Cover frosted glass & $\begin{array}{l}\text { Cover } \\
\text { clear glass }\end{array}$ & Flat glass \\
\hline LED Module & $\begin{array}{l}\text { LED High Power } 18 \\
\text { LEDs }\end{array}$ & $\begin{array}{l}\text { LED High Power } 18 \\
\text { LEDs }\end{array}$ & $\begin{array}{l}\text { LED MidPower } 30 \\
\text { LEDs }\end{array}$ & $\begin{array}{l}\text { LED High Power } \\
12 \text { LEDs }\end{array}$ \\
\hline Lantern luminous flux & $3000 \mathrm{~lm}$ & $2500 \mathrm{~lm}$ & $2500 \mathrm{~lm}$ & $2000 \mathrm{~lm}$ \\
\hline Luminous efficiency & $70 \mathrm{~lm} / \mathrm{W}$ & $79 \mathrm{~lm} / \mathrm{W}$ & $84 \mathrm{~lm} / \mathrm{W}$ & $85 \mathrm{~lm} / \mathrm{W}$ \\
\hline $\begin{array}{l}\text { Average illuminance } \\
\text { in condition } 1 \mathrm{~S} \\
\text { [min;max] }\end{array}$ & 12 lx [2 lx; 57 lx] & 11 1x [2 1x; $281 x]$ & $111 x$ [4 1x; $401 x]$ & $\begin{array}{l}12 \operatorname{lx}[21 \mathrm{~lx} ; 34 \\
1 \mathrm{x}]\end{array}$ \\
\hline $\begin{array}{l}\text { Average illuminance } \\
\text { in condition } 2 \mathrm{~S} \\
\text { [min;max] }\end{array}$ & 13 lx [3 lx; 58 lx] & 13 lx [3 lx; $291 x]$ & 14 lx [7 1x; $421 x]$ & $\begin{array}{l}14 \text { lx [3 1x; } 34 \\
1 x]\end{array}$ \\
\hline
\end{tabular}


Table 3. Characteristics of the panel

\begin{tabular}{lll}
\hline & & Panel (\%) \\
\hline Gender & Male & 42 \\
& Female & 58 \\
\hline Age & $<35$ & 33.3 \\
& $35-50$ & 33.3 \\
& $>50$ & 33.3 \\
\hline Corrected vision & Yes & 60 \\
& No & 40 \\
\hline Visual acuity [Mesopic in brackets] & $12 / 10$ & $70[0]$ \\
& $\geq 10 / 10$ & $79[6]$ \\
& $\geq 8 / 10$ & $94[46]$ \\
& $\geq 6 / 10$ & $94[70]$ \\
& $\geq 5 / 10$ & $100[70]$ \\
& $\geq 4 / 10$ & $100[97]$ \\
& $\geq 2 / 10$ & $100[100]$ \\
\hline Contrast sensitivity & Very good (0 err.) & 48 \\
& Good (1-2 err.) & 12 \\
& Medium (3-4 err.) & 3 \\
& Bad (5-9 err.) & 15 \\
& Very Bad (>10 err.) & 21 \\
\hline Recovery time after glare & $<25$ s & 61 \\
& $25-50$ s & 33 \\
& $>50$ s & 6 \\
\hline
\end{tabular}


Table 4. Photometric characteristics of the stimuli

\begin{tabular}{|c|c|c|c|c|c|c|c|c|c|c|c|c|c|c|c|}
\hline \multirow[b]{2}{*}{ Lantern } & \multirow[b]{2}{*}{ Position } & \multirow[b]{2}{*}{ Direction } & \multicolumn{2}{|c|}{ Eccentricity } & \multicolumn{2}{|c|}{ LMT B520 } & \multicolumn{5}{|c|}{ Konica-Minolta CA-2000A } & \multicolumn{4}{|c|}{ Canon EO } \\
\hline & & & $\begin{array}{c}\theta \\
\text { Lum1 } \\
\left({ }^{\circ}\right)\end{array}$ & $\begin{array}{c}\theta \\
\operatorname{Lum} 2 \\
\left(^{\circ}\right)\end{array}$ & $\begin{array}{c}\text { Etot }(1 \mathrm{x}) \\
1 \mathrm{~S}\end{array}$ & $\begin{array}{c}\text { Etot }(\mathrm{lx}) \\
2 \mathrm{~S}\end{array}$ & $\begin{array}{c}\mathrm{L}_{\mathrm{RZ}} \\
\left(\mathrm{cd} / \mathrm{m}^{2}\right) \\
1 \mathrm{~S}\end{array}$ & $\begin{array}{c}\mathrm{L}_{\mathrm{DZ}} \\
\left(\mathrm{cd} / \mathrm{m}^{2}\right) \\
1 \mathrm{~S}\end{array}$ & $\begin{array}{c}\mathrm{L}_{\mathrm{RZ}} \\
\left(\mathrm{cd} / \mathrm{m}^{2}\right) \\
2 \mathrm{~S}\end{array}$ & $\begin{array}{c}\mathrm{L}_{\mathrm{DZ}} \\
\left(\mathrm{cd} / \mathrm{m}^{2}\right) \\
2 \mathrm{~S}\end{array}$ & $\mathrm{E}_{\text {black }}(\mathrm{lx})$ & $\begin{array}{c}\mathrm{L}_{\mathrm{LED}} \\
\left(\mathrm{cd} / \mathrm{m}^{2}\right) \\
\mathrm{Lum} 1\end{array}$ & $\begin{array}{c}\mathrm{L}_{\mathrm{LED}} \\
\left(\mathrm{cd} / \mathrm{m}^{2}\right) \\
\mathrm{Lum} 2\end{array}$ & $\begin{array}{c}\mathrm{L}_{\text {Lantern }} \\
\left(\mathrm{cd} / \mathrm{m}^{2}\right) \\
\text { Lum1 }\end{array}$ & $\begin{array}{c}\mathrm{L}_{\text {Lante }} \\
(\mathrm{cd} / \mathrm{m} \\
\text { Lum }\end{array}$ \\
\hline \multirow{8}{*}{ A } & \multirow{2}{*}{$8.5 \mathrm{~m}$} & $0^{\circ}$ & 22.6 & 9.2 & 5.2 & 5.6 & 0.197 & 0.096 & 0.349 & 0.247 & \multirow{8}{*}{$\begin{array}{l}0.050 \\
0.060 \\
0.047 \\
0.060 \\
0.047 \\
0.053 \\
0.050 \\
0.053\end{array}$} & & & & \\
\hline & & $10^{\circ}$ & 30.4 & 17.7 & 4.8 & 5.1 & 0.113 & 0.056 & 0.205 & 0.12 & & & & & r \\
\hline & \multirow{2}{*}{$6.5 \mathrm{~m}$} & $0^{\circ}$ & 28.5 & 10.1 & 7.9 & 8.4 & 0.144 & 0.055 & 0.32 & 0.217 & & \multirow{2}{*}{$1.29 \mathrm{E}+04$} & \multirow{2}{*}{$6.00 \mathrm{E}+03$} & \multirow{2}{*}{$4.10 \mathrm{E}+03$} & \multirow{2}{*}{$1.98 \mathrm{E}$} \\
\hline & & $10^{\circ}$ & 36.3 & 18.6 & 7.1 & 7.7 & 0.099 & 0.05 & 0.211 & 0.122 & & & & & \\
\hline & \multirow{2}{*}{$4.5 \mathrm{~m}$} & $0^{\circ}$ & 38.2 & 11.2 & 12.3 & 13.1 & 0.108 & 0.042 & 0.317 & 0.214 & & \multirow{2}{*}{$1.30 \mathrm{E}+04$} & \multirow{2}{*}{$7.70 \mathrm{E}+03$} & \multirow{2}{*}{$3.90 \mathrm{E}+03$} & \multirow{2}{*}{2.171} \\
\hline & & $10^{\circ}$ & 45.7 & 19.6 & 10.7 & 11.5 & 0.085 & 0.043 & 0.226 & 0.124 & & & & & \\
\hline & \multirow{2}{*}{$2.5 \mathrm{~m}$} & $0^{\circ}$ & 54.7 & 12.6 & 15.5 & 16.2 & 0.072 & 0.034 & 0.308 & 0.206 & & \multirow{2}{*}{$1.10 \mathrm{E}+04$} & \multirow{2}{*}{$9.80 \mathrm{E}+03$} & \multirow{2}{*}{$4.30 \mathrm{E}+03$} & \\
\hline & & $10^{\circ}$ & 62.1 & 20.9 & 11.2 & 12.7 & 0.066 & 0.044 & 0.226 & 0.129 & & & & & 2.971 \\
\hline & & $0^{\circ}$ & 22.6 & 9.2 & 5.5 & 5.7 & 0.221 & 0.117 & 0.341 & 0.232 & 0.047 & & & & \\
\hline & & $10^{\circ}$ & 30.4 & 17.7 & 5.0 & 5.3 & 0.116 & 0.068 & 0.188 & 0.12 & 0.057 & & & & \\
\hline & $65 \mathrm{~m}$ & $0^{\circ}$ & 28.5 & 10.1 & 8.3 & 8.7 & 0.171 & 0.071 & 0.315 & 0.199 & 0.050 & & & & 50 \\
\hline & & $10^{\circ}$ & 36.3 & 18.6 & 7.6 & 7.8 & 0.115 & 0.065 & 0.205 & 0.124 & 0.057 & & & & \\
\hline & & $0^{\circ}$ & 38.2 & 11.2 & 12.5 & 13.0 & 0.131 & 0.051 & 0.309 & 0.183 & 0.047 & & & & \\
\hline & & $10^{\circ}$ & 45.7 & 19.6 & 10.7 & 11.3 & 0.104 & 0.059 & 0.216 & 0.122 & 0.053 & 04 & & SJ & 17 \\
\hline & $25 \mathrm{~m}$ & $0^{\circ}$ & 54.7 & 12.6 & 13.8 & 14.4 & 0.099 & 0.044 & 0.297 & 0.174 & 0.047 & & & & 81 \\
\hline & & $10^{\circ}$ & 62.1 & 20.9 & 10.6 & 11.6 & 0.089 & 0.058 & 0.214 & 0.124 & 0.053 & & (נת & 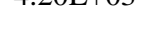 & \\
\hline & & $0^{\circ}$ & 22.6 & 9.2 & 20.9 & 22.3 & 0.314 & 0.237 & 0.637 & 0.542 & 0.057 & & & & \\
\hline & & $10^{\circ}$ & 30.4 & 17.7 & 19.8 & 21.0 & 0.159 & 0.104 & 0.334 & 0.205 & 0.082 & 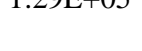 & & 504 & .21 \\
\hline & & $0^{\circ}$ & 28.5 & 10.1 & 24.9 & 26.5 & 0.207 & 0.11 & 0.577 & 0.454 & 0.057 & & & 04 & 71 \\
\hline C & & $10^{\circ}$ & 36.3 & 18.6 & 22.6 & 25.9 & 0.148 & 0.1 & 0.376 & 0.22 & 0.079 & & & & \\
\hline & & $0^{\circ}$ & 38.2 & 11.2 & 22.5 & 25.6 & 0.161 & 0.08 & 0.603 & 0.442 & 0.060 & & & & \\
\hline & II & $10^{\circ}$ & 45.7 & 19.6 & 20.0 & 22.7 & 0.144 & 0.098 & 0.436 & 0.238 & 0.082 & PUT & 74 & $0 J$ & 5.00 \\
\hline & & $0^{\circ}$ & 54.7 & 12.6 & 15.4 & 19.6 & 0.144 & 0.069 & 0.604 & 0.441 & 0.063 & & 04 & +03 & \\
\hline & & $10^{\circ}$ & 62.1 & 20.9 & 12.1 & 16.7 & 0.139 & 0.096 & 0.443 & 0.234 & 0.079 & & . & o & \\
\hline & & $0^{\circ}$ & 22.6 & 9.2 & 6.8 & 6.6 & 0.257 & 0.145 & 0.383 & 0.271 & 0.057 & & & & \\
\hline & & $10^{\circ}$ & 30.4 & 17.7 & 6.0 & 6.2 & 0.14 & 0.079 & 0.214 & 0.129 & 0.085 & & & & \\
\hline & $6.5 \mathrm{~m}$ & $0^{\circ}$ & 28.5 & 10.1 & 16.0 & 16.1 & 0.205 & 0.083 & 0.367 & 0.222 & 0.060 & $16 \mathrm{E}+05$ & $570 \mathrm{E}+02$ & $293 \mathrm{E}+04$ & 87 \\
\hline & & $10^{\circ}$ & 36.3 & 18.6 & 14.6 & 14.7 & 0.14 & 0.073 & 0.241 & 0.127 & 0.085 & & & & \\
\hline & & $0^{\circ}$ & 38.2 & 11.2 & 21.8 & 22.6 & 0.165 & 0.06 & 0.365 & 0.21 & 0.060 & 05 & & & \\
\hline & & $10^{\circ}$ & 45.7 & 19.6 & 20.5 & 20.2 & 0.128 & 0.067 & 0.258 & 0.133 & 0.079 & & 2 & 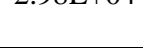 & \\
\hline & $2.5 \mathrm{~m}$ & $0^{\circ}$ & 54.7 & 12.6 & 21.3 & 22.4 & 0.116 & 0.042 & 0.346 & 0.181 & 0.060 & $1.13 \mathrm{E}+05$ & $9.00 \mathrm{E}+02$ & $1.94 \mathrm{E}+04$ & 480 \\
\hline & & $10^{\circ}$ & 62.1 & 20.9 & 18.3 & 20.0 & 0.103 & 0.061 & 0.252 & 0.124 & 0.085 & & & & \\
\hline
\end{tabular}



Table 5. Mean values of the glare ratings in the different experimental conditions (standard deviation values are in brackets).

\begin{tabular}{|l|l|l|l|l|}
\hline Factor & Lantern & $\begin{array}{l}\text { Number of } \\
\text { luminaires }\end{array}$ & $\begin{array}{l}\text { Gaze } \\
\text { direction }\end{array}$ & Position \\
\hline Static & A: $6.9[1.6]$ & 1S: $6.2[2.0]$ & $0^{\circ}: 6.3[2.0]$ & $8.5 \mathrm{~m}: 6.5[2.1]$ \\
Protocol & B: $6.8[1.7]$ & 2S: $6.5[1.9]$ & $10^{\circ}: 6.5[1.9]$ & $6.5 \mathrm{~m}: 6.2[2.0]$ \\
& C: $5.6[2.1]$ & & & $4.5 \mathrm{~m}: 6.2[1.9]$ \\
& D: $6.2[2.1]$ & & & $2.5 \mathrm{~m}: 6.5[1.8]$ \\
\hline Dynamic & A: $7.4[1.5]$ & 1S: $6.9[1.8]$ & & \\
Protocol & B: $7.1[1.6]$ & 2S: $6.7[1.8]$ & & \\
& C: $5.9[1.9]$ & & & \\
& D: $6.7[1.7]$ & & & \\
\hline
\end{tabular}


Table 6. Prediction comparisons: RMSE and Spearman correlation $\mathrm{R}^{2}$ between predicted scores on the de Boer scale and mean ratings in the "Static" experiment, in condition $1 \mathrm{~S}$

\begin{tabular}{lllrc}
\hline Model & Background & Source & RMSE & $\mathrm{R}^{\mathbf{2}}$ \\
\hline Sch74 & $\mathrm{L}_{\mathrm{RZ}}$ & & 4.40 & 0.744 \\
Sch74 & $\mathrm{L}_{\mathrm{DZ}}$ & & 4.57 & 0.751 \\
\hline Lin14 & $\mathrm{L}_{\mathrm{RZ}}$ & $\mathrm{L}_{\text {Lantern }}$ & 3.94 & 0.384 \\
Lin14 & $\mathrm{L}_{\mathrm{DZ}}$ & $\mathrm{L}_{\text {Lantern }}$ & 4.23 & 0.370 \\
Lin14 & $\mathrm{L}_{\mathrm{RZ}}$ & $\mathrm{L}_{\mathrm{LED}}$ & 3.76 & 0.375 \\
Lin14 & $\mathrm{L}_{\mathrm{DZ}}$ & $\mathrm{L}_{\mathrm{LED}}$ & 4.05 & 0.368 \\
\hline Lin15 & $\mathrm{E}_{\mathrm{RZ}}$ & & 0.81 & 0.781 \\
Lin15 & $\mathrm{E}_{\mathrm{DZ}}$ & & 0.60 & 0.745 \\
\hline Bul11 & $\mathrm{E}_{\mathrm{s}}=\mathrm{E}_{\mathrm{tot}}-\mathrm{E}_{\mathrm{l}}-\mathrm{E}_{\mathrm{a}}$ & & 1.27 & 0.648 \\
Bul11 & $\mathrm{E}_{\mathrm{s}}=\mathrm{E}_{\mathrm{RZ}}$ & & 1.13 & 0.720 \\
Bul11 & $\mathrm{E}_{\mathrm{s}}=\mathrm{E}_{\mathrm{DZ}}$ & & 1.30 & 0.725 \\
\hline
\end{tabular}


Table 7. RMSE and $\mathrm{R}^{2}$ between the predictions computed with the simplified method and the mean glare ratings collected in Condition $2 \mathrm{~S}$.

\begin{tabular}{lllll}
\hline Model & Background & Source & $\begin{array}{l}\text { RMSE } \\
\text { with mean } \\
\text { ratings }\end{array}$ & $\begin{array}{l}\mathrm{R}^{2} \\
\text { Spearman } \\
\text { with mean } \\
\text { ratings }\end{array}$ \\
\hline Sch74 & $\mathrm{L}_{\mathrm{RZ}}$ & & 4.46 & 0.812 \\
Sch74 & $\mathrm{L}_{\mathrm{DZ}}$ & & 4.61 & 0.796 \\
\hline Lin14 & $\mathrm{L}_{\mathrm{RZ}}$ & $\mathrm{L}_{\text {Lantern }}$ & 3.87 & 0.566 \\
Lin14 & $\mathrm{L}_{\mathrm{DZ}}$ & $\mathrm{L}_{\mathrm{Lantern}}$ & 4.09 & 0.512 \\
Lin14 & $\mathrm{L}_{\mathrm{RZ}}$ & $\mathrm{L}_{\mathrm{LED}}$ & 3.69 & 0.543 \\
Lin14 & $\mathrm{L}_{\mathrm{DZ}}$ & $\mathrm{L}_{\mathrm{LED}}$ & 3.90 & 0.486 \\
\hline Lin15 & $\mathrm{E}_{\mathrm{RZ}}$ & & 0.87 & 0.745 \\
Lin15 & $\mathrm{E}_{\mathrm{DZ}}$ & & 0.70 & 0.733 \\
\hline Bu111 & $\mathrm{E}_{\mathrm{s}}=\mathrm{E}_{\mathrm{tot}}-\mathrm{E}_{\mathrm{l}}-\mathrm{E}_{\mathrm{a}}$ & & 1.42 & 0.740 \\
Bul11 & $\mathrm{E}_{\mathrm{s}}=\mathrm{E}_{\mathrm{RZ}}$ & & 1.22 & 0.795 \\
Bul11 & $\mathrm{E}_{\mathrm{s}}=\mathrm{E}_{\mathrm{DZ}}$ & & 1.35 & 0.787 \\
\hline
\end{tabular}



Table 8. RMSE and $\mathrm{R}^{2}$ between the Dir/Ind predictions and the mean glare ratings collected in Condition 1S and Condition 2S (simplified method).

\begin{tabular}{llll}
\hline Condition & Background & $\begin{array}{l}\text { RMSE with } \\
\text { mean ratings }\end{array}$ & $\begin{array}{l}\text { Spearman } \mathrm{R}^{2} \\
\text { with mean } \\
\text { ratings }\end{array}$ \\
\hline Condition 1S & $\mathrm{E}_{\mathrm{RZ}}$ & 0.76 & 0.655 \\
& $\mathrm{E}_{\mathrm{DZ}}$ & 1.05 & 0.676 \\
\hline Condition 2S & $\mathrm{E}_{\mathrm{RZ}}$ & 0.84 & 0.655 \\
& $\mathrm{E}_{\mathrm{DZ}}$ & 0.60 & 0.688 \\
\hline
\end{tabular}

